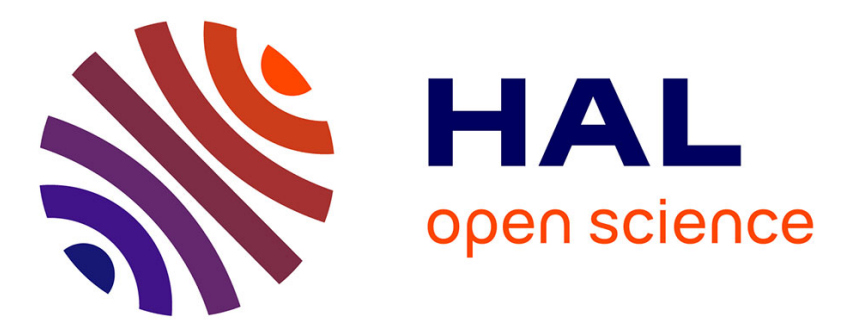

\title{
A consistent methodology for the derivation and calibration of a macroscopic turbulence model for flows in porous media
}

Marie Drouin, Olivier Grégoire, Olivier Simonin

\section{- To cite this version:}

Marie Drouin, Olivier Grégoire, Olivier Simonin. A consistent methodology for the derivation and calibration of a macroscopic turbulence model for flows in porous media. International Journal of Heat and Mass Transfer, 2013, vol. 63, pp. 401-413. 10.1016/j.ijheatmasstransfer.2013.03.060 . hal00952032

\section{HAL Id: hal-00952032 \\ https://hal.science/hal-00952032}

Submitted on 26 Feb 2014

HAL is a multi-disciplinary open access archive for the deposit and dissemination of scientific research documents, whether they are published or not. The documents may come from teaching and research institutions in France or abroad, or from public or private research centers.
L'archive ouverte pluridisciplinaire HAL, est destinée au dépôt et à la diffusion de documents scientifiques de niveau recherche, publiés ou non, émanant des établissements d'enseignement et de recherche français ou étrangers, des laboratoires publics ou privés. 




\section{Open Archive TOULOUSE Archive Ouverte (OATAO)}

OATAO is an open access repository that collects the work of Toulouse researchers and makes it freely available over the web where possible.

This is an author-deposited version published in : http://oatao.univ-toulouse.fr/ Eprints ID : 11035

To link to this article : DOI: 10.1016/j.ijheatmasstransfer.2013.03.060 http://dx.doi.org/10.1016/j.ijheatmasstransfer.2013.03.060

To cite this version : Drouin, Marie and Grégoire, Olivier and Simonin, Olivier A consistent methodology for the derivation and calibration of a macroscopic turbulence model for flows in porous media. (2013) International Journal of Heat and Mass Transfer, vol. 63 .pp. 401-413. ISSN 0017-9310

Any correspondance concerning this service should be sent to the repository administrator: staff-oatao@,1istes-diff.inp-toulouse.fr 


\title{
A consistent methodology for the derivation and calibration of a macroscopic turbulence model for flows in porous media
}

\author{
M. Drouin ${ }^{\mathrm{b}}$, O. Grégoire ${ }^{\mathrm{a}, *}$, O. Simonin ${ }^{\mathrm{c}}$ \\ a STXN, 25 rue Leblanc, 75015 Paris, France \\ ${ }^{\mathrm{b}}$ CEA Saclay, DEN/DANS/DM2S/STMF/LATF, 91191 Gif-sur-Yvette, France \\ ${ }^{\mathrm{c}}$ Université de Toulouse, CNRS IMFT, Allée du Professeur Camille Soula, 31400 Toulouse, France
}

Keywords:

Macroscopic turbulence model

Double averaging

Turbulence

Porous media

Dispersion

\begin{abstract}
A B S T R A C T
This work aims to model turbulent flows in media laden with solid structures according to porous media approach. A complete set of macroscopic transport equations is derived by spatially averaging the Reynolds averaged governing equations. A two-scale analysis highlights energy transfers between macroscopic and sub-filter kinetic energies (dispersive and turbulent kinetic energies). Additional terms coming from the averaging procedure and representing solids/fluid interactions and turbulent contributions are modeled. Connections between turbulence modeling and dispersion modeling are presented. Other closure expressions are determined using physical considerations and spatial averaging of microscopic computations. A special care is given to the calibration methodology for the phenomenological coefficients. Results of the present model are successfully compared to volume-averaged reference results coming from fine scale computations and show significant improvements with respect to previous macroscopic models.
\end{abstract}

\section{Introduction}

Due to their size and complexity, large industrial devices are not easy to model and calculate. They generally combine free flow regions connected with regions densely occupied with solids (plates, rod bundles, ...). For such devices, fine scale simulations are still unachievable and even if they were, they would produce, at a very expensive cost, plenty of data irrelevant for engineering purposes (design, safety analysis, ...). This is the reason why most engineers use homogenized computation codes for the calculation of media densely laden with solid structures (say the core region for a nuclear reactor for instance). Fine scale simulations are well adapted for (nearly) free flow regions and homogenized models are doing a rather good job in the densely laden regions (say porous regions).

On the other hand, fine scale simulations are now accurate enough to recover most of the details of the flow at the sub-channel (or Representative Elementary Volume - REV) scale of the porous region and to produce "reference results" at this scale. According to this statement, many researchers aim to derive macroscopic turbulence model for media laden with solid structures through upscaling of fine scale results. Most attempts to model turbulent flows in porous media are based on methods usually followed to model turbulence in clear flows. Not surprisingly, the most com-

\footnotetext{
* Corresponding author. Tel.: +33 164502743.

E-mail address: olivier.gregoire@cea.fr (O. Grégoire).
}

mon model for macroscopic turbulence available in the literature is a $\bar{k}-\bar{\varepsilon}$ adapted to porous media $[1,12,16]$. It is obtained by spatially averaging (averaging operators are introduced in Section 2) the classical two-equations $\bar{k}-\bar{\varepsilon}$ model:

$$
\begin{aligned}
\phi \frac{\partial\langle\bar{k}\rangle_{f}}{\partial t}+\frac{\partial}{\partial x_{i}}\left(\phi\left\langle\bar{u}_{i}\right\rangle_{f}\langle\bar{k}\rangle_{f}\right)= & \frac{\partial}{\partial x_{j}}\left(\left[v_{f}+\frac{v_{t \phi}}{\sigma_{k}}\right] \frac{\partial \phi\langle\bar{k}\rangle_{f}}{\partial x_{j}}\right) \\
& +2 \phi v_{t \phi}\left\langle s_{i j}\right\rangle_{f}\left\langle s_{i j}\right\rangle_{f}-\phi\langle\bar{\varepsilon}\rangle_{f}+\phi P_{S F}, \\
\phi \frac{\partial\langle\bar{\varepsilon}\rangle_{f}}{\partial t}+\frac{\partial}{\partial x_{i}}\left(\phi\left\langle\bar{u}_{i}\right\rangle_{f}\langle\bar{\varepsilon}\rangle_{f}\right)= & \frac{\partial}{\partial x_{j}}\left(\left[v_{f}+\frac{v_{t \phi}}{\sigma_{\epsilon}}\right] \frac{\partial \phi\langle\bar{k}\rangle_{f}}{\partial x_{j}}\right) \\
& +2 \frac{C_{\varepsilon_{1}}}{\tau_{d}} \phi v_{t \phi}\left\langle s_{i j}\right\rangle_{f}\left\langle s_{i j}\right\rangle_{f}-\frac{C_{\varepsilon_{2}}}{\tau_{d}} \phi\langle\bar{\varepsilon}\rangle_{f} \\
& +\frac{C_{\varepsilon_{2}}}{\tau_{p}} \phi P_{S F} .
\end{aligned}
$$

In the previous equations, $P_{S F}$ stands for the spatially averaged turbulence production at the sub-filter scale and is called "sub-filter production". Chandesris et al. [1], Nakayama and Kuwahara [12] and Pedras and de Lemos [16] models mainly differ from each other by the definition of sub-filter production, and time scale $\tau_{p}$ while they all use the same definition for the dissipation time scale $\tau_{d}$. A comparison of the various source terms of these models is summarized in [4] (see Table 9.1, p. 143). 
Thanks to a two-scale analysis, Pinson et al. [18] exhibit energy transfers between kinetic energies at different scales and highlight the role of the wake dissipation $\left(\left\langle\bar{\varepsilon}_{w}\right\rangle_{f}\right)$. In canopy turbulence models, Green [8] considers that the sub-filter production is the product between drag and the mean macroscopic velocity minus a sink term that represents the accelerated cascade of turbulent kinetic energy due to the plant foliage. Considering that the wake dissipation is linked to the sub-filter mean kinetic energy, Pinson [17] postulates an additional transport equation for $\left\langle\bar{\varepsilon}_{w}\right\rangle_{f}$

$\frac{\partial\left\langle\bar{\varepsilon}_{w}\right\rangle_{f}}{\partial t}+\left\langle\bar{u}_{z}\right\rangle_{f} \frac{\partial\left\langle\bar{\varepsilon}_{w}\right\rangle_{f}}{\partial z}=\frac{C_{w}}{\tau_{p}}\left\langle\bar{u}_{z}\right\rangle_{f} \bar{F}_{\phi_{z}}-\frac{C_{w}}{\tau_{w}}\left\langle\bar{\varepsilon}_{w}\right\rangle_{f}$,

that completes the $\langle\bar{k}\rangle_{f}-\langle\bar{\varepsilon}\rangle_{f}$ system. Assuming that the sub-filter mean kinetic energy (or "dispersive kinetic energy", $\left.\left\langle\bar{E}^{m}\right\rangle_{f}=\frac{1}{2}\left\langle\delta \bar{u}_{i} \delta \bar{u}_{i}\right\rangle_{f}\right)$ remains nearly constant for homogeneous fully developed flows with no macroscopic shear, Pinson et al. [18] obtain the following algebraic closure for sub-filter production:

$P_{S F}=\left\langle\bar{u}_{z}\right\rangle_{f} \bar{F}_{\phi_{z}}-\left\langle\bar{\varepsilon}_{w}\right\rangle_{f}$.

The model of Pinson et al. [18] gives a much better representation of the dynamic behavior of the flow than the ones of Nakayama and Kuwahara [12], Pedras and de Lemos [16] and Chandesris et al. [1]. However, since then we have experienced that closure (4) is questionable. Indeed, $\left\langle\bar{E}^{m}\right\rangle_{f}$ may undergo large departure from equilibrium on a wide region of the flow.

Teruel and Rizwan-uddin [21,22] propose to directly model the entire kinetic energy filtered in the averaging process. To this aim, they define a double averaging operator, denoted $\bar{\xi}$, such that any quantity $\xi$ can then be decomposed into a space-time averaged quantity and a fluctuation $\xi=\bar{\xi}+\xi^{\prime \prime}$. This operator is idempotent under the separation of scales hypothesis. Then, they define a macroscopic turbulent kinetic energy (MTKE) that includes both dispersive and turbulent kinetic energies

$\overline{\bar{k}}=\frac{1}{2} \overline{\overline{u_{i}^{\prime \prime} u_{i}^{\prime \prime}}}=\left\langle\bar{E}^{m}\right\rangle_{f}+\langle\bar{k}\rangle_{f}$

and model its balance equation. Doing so, they do not neglect anymore dispersive kinetic energy variations, a contrario with other models. They also define the macroscopic turbulent dissipation rate (MTDR) which accounts for viscous and wake dissipation

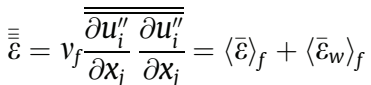

and postulate its modeled transport equation. The model of Teruel and Rizwan-uddin [21] gives good results for $\overline{\bar{k}}$ and $\overline{\bar{\varepsilon}}$ and accounts for all the information lost during the averaging procedure. Nevertheless, no distinction is made between dispersive and turbulent effects and thus highly unbalanced configurations can not be properly calculated.

In this paper, we propose the derivation and calibration of a practical macroscopic turbulent model that aims to:

- faithfully recover the fully developed flow limit,

- capture dynamical behavior for major quantities submitted to perturbations,

- provide relevant boundary conditions for turbulent quantities and thus is able to exchange with free flow regions,

- connect dispersion modeling with turbulence modeling [9].

Thanks to the two scale analysis, Pinson et al. [18] have shown that before it is degraded into turbulence, mean motion kinetic energy first supplies the so-called dispersive kinetic energy through the sub-filter production. When flows undergo large perturbations (for instance at the inlet/outlet of porous region), dispersive kinetic energy and turbulent kinetic energy reach large unbalances with respect to their equilibrium values. This results in variations of the friction factor and heat exchange coefficient. In order to analyze these phenomenological features, we derive in Section 2 a complete set of spatially averaged governing equations for macroscopic mean kinetic energy, dispersive kinetic energy and averaged turbulent kinetic energy. All transfer modes between those kinetic energies are then highlighted. Combined with the analysis of numerous reference results, this analysis of transfers leads us to model three transport equations for turbulent kinetic energy, wake dissipation and, for the first time, dispersive kinetic energy. A priori tests have shown that turbulent dissipation $\left(\langle\bar{\varepsilon}\rangle_{f}\right)$ might be easily fit by a simple correlation. Consequently, this equation is dropped and replaced by a correlation. On the other hand, an equation for the dispersive kinetic energy is derived in order to capture the dynamics of the flow at the subfilter scale.

Closures for the transport equations are detailed in Section 3. A rigorous calibration methodology based upon both asymptotic state and perturbation analysis is then proposed in Section 4 for model coefficients. Finally, in order to fix the unknown model coefficients, we perform a priori tests using spatially averaged fine scale simulation results [3]. The macroscopic turbulence model is then successfully tested for the case of unidirectional turbulent flows through stratified porous media. Improvements with respect to previous modelizations are highlighted.

\section{Derivation of governing equations and transfer analysis}

For turbulent flows in porous media, two averaging operators are generally used: a statistical average, that is practical to handle turbulence, and a spatial average, well adapted to the porous media approach [24]. Pedras and de Lemos [16] have shown that both averages commute in a strict mathematical point of view. However, the macroscopic turbulence modelization necessarily depends on the order of application of these operators as discussed by Nield [14] and Travkin [23]. Following Pedras and de Lemos [16], Pinson et al. [18], Chandesris et al. [1], Masuoka and Takatsu [11], Nakayama and Kuwahara [12], we apply a volume-average operator to the local statistically averaged equations. Reynolds Averaged Navier-Stokes (RANS) equations are spatially averaged in order to get a structured picture of the turbulent flow that allows to benefit from the large amount of knowledge available for (RANS) turbulence modeling (see Fig. 1).

In this paper, uncompressible, single phase flows in saturated, rigid porous media are considered. Fluid properties (density, viscosity) are assumed constant. The Reynolds-averaged set of governing equations, namely the continuity equation, the momentum equation and the turbulent kinetic energy equation, are then given by

$\frac{\partial \bar{u}_{i}}{\partial x_{i}}=0$,

$\frac{\partial \bar{u}_{i}}{\partial t}+\frac{\partial \bar{u}_{i} \bar{u}_{j}}{\partial x_{j}}=-\frac{1}{\rho} \frac{\partial \bar{P}}{\partial x_{i}}+\frac{\partial}{\partial x_{j}}\left(v_{f} \frac{\partial \bar{u}_{i}}{\partial x_{j}}\right)-\frac{\partial \overline{u_{i}^{\prime} u_{j}^{\prime}}}{\partial x_{j}}$

$\frac{\partial \bar{k}}{\partial t}+\frac{\partial \bar{u}_{i} \bar{k}}{\partial x_{i}}=-\frac{\partial}{\partial x_{i}} \overline{\left(\frac{P^{\prime}}{\rho}+k^{\prime}\right) u_{i}^{\prime}}+\frac{\partial}{\partial x_{i}}\left(v_{f} \frac{\partial \bar{k}}{\partial x_{i}}\right)-\overline{u_{i}^{\prime} u_{j}^{\prime}} \frac{\partial \bar{u}_{i}}{\partial x_{j}}$ $-v_{f} \frac{\overline{\partial u_{i}^{\prime}}}{\partial x_{j}} \frac{\partial u_{i}^{\prime}}{\partial x_{j}}$ 


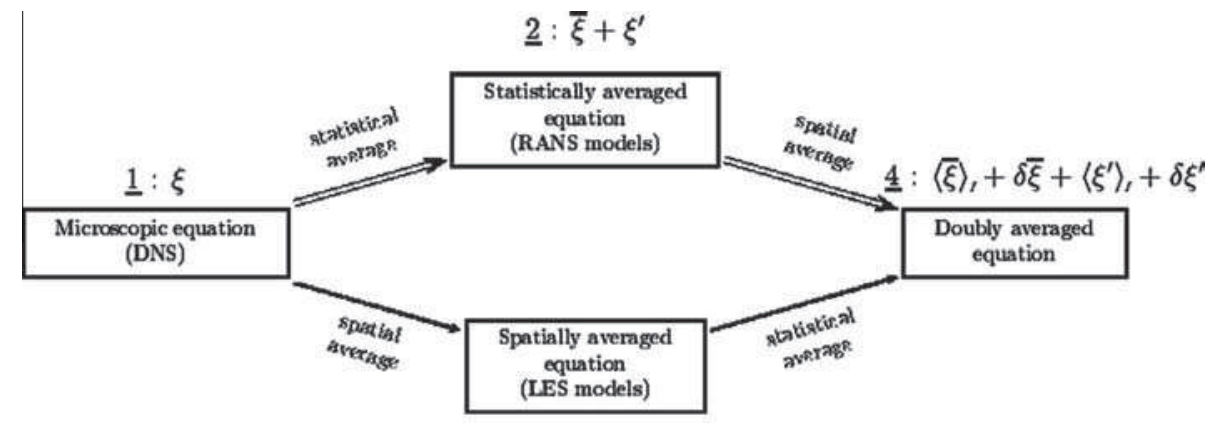

$\underline{3}:\langle\xi\rangle_{t}+\delta \xi$

Fig. 1. Averaging procedure: order of application of the filters.

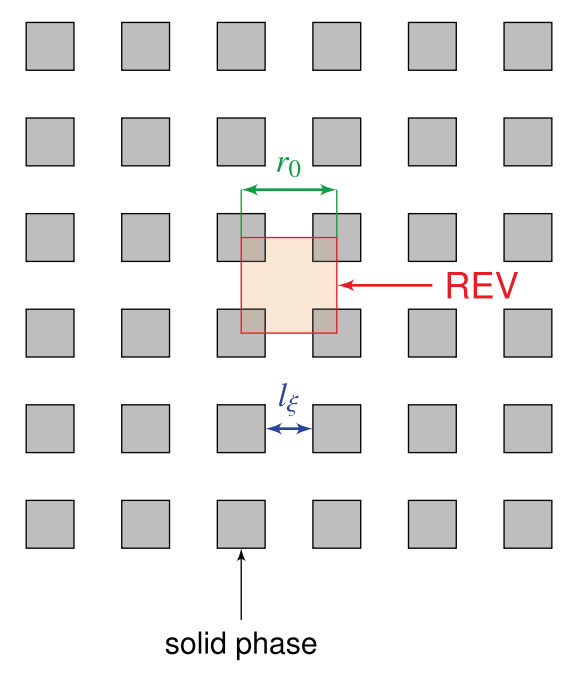

(a) Ordered porous media

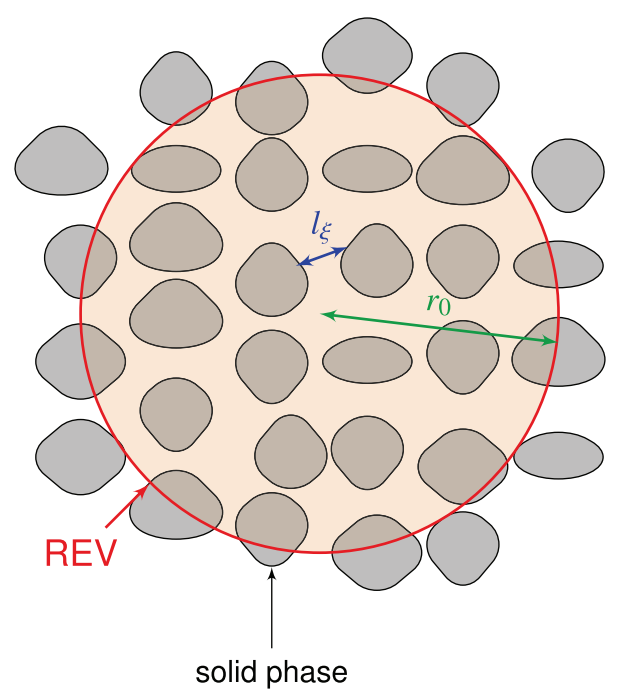

(b) Disordered porous media

Fig. 2. Representative Elementary Volume in ordered and disordered porous media.

Following "the upper way" exposed in Fig. 1, RANS model equations are integrated over a Representative Elementary Volume $\Delta V$, which is assumed to be well adapted to the geometrical characteristics of the media under study [20]. The spatial average is then defined by
$\langle\xi\rangle_{f}(\mathbf{x})=\frac{1}{\Delta V_{f}(\mathbf{x})} \int_{\Delta V_{f}(\mathbf{x})} \xi d V$

where $\Delta V_{f}$ is the volume of fluid embedded within $\Delta V$ (see Fig. 2). The ratio of the fluid volume on the total volume defines porosity (hereafter denoted $\phi$ ).

The spatial average can be assumed idempotent if variation length scales of the macroscopic quantities are large with respect to the filter size [20]. For each average, any quantity $\xi$ may be split into mean and fluctuating components as

$\xi=\bar{\xi}+\xi^{\prime}=\langle\xi\rangle_{f}+\delta \xi$,

and one can write

$\xi=\langle\bar{\xi}\rangle_{f}+\left\langle\xi^{\prime}\right\rangle_{f}+\delta \bar{\xi}+\delta \xi^{\prime}$.

No-slip condition at wall and properties of the spatial average operator [5] allow us to write the doubly averaged mass conservation

$\phi\left\langle\frac{\partial \bar{u}_{i}}{\partial x_{i}}\right\rangle_{f}=\frac{\partial \phi\left\langle\bar{u}_{i}\right\rangle_{f}}{\partial x_{i}}=0$,

and the macroscopic momentum equation

$$
\begin{aligned}
\phi \frac{\partial\left\langle\bar{u}_{i}\right\rangle_{f}}{\partial t}+\frac{\partial \phi\left\langle\bar{u}_{i}\right\rangle_{f}\left\langle\bar{u}_{j}\right\rangle_{f}}{\partial x_{j}}= & -\frac{1}{\rho} \frac{\partial \phi\langle\bar{P}\rangle_{f}}{\partial x_{i}}+\frac{\partial}{\partial x_{j}}\left(v_{f} \frac{\partial \phi\left\langle\bar{u}_{i}\right\rangle_{f}}{\partial x_{j}}\right) \\
& -\frac{\partial \phi\left\langle\overline{\left.u_{i}^{\prime} \bar{u}_{j}^{\prime}\right\rangle_{f}}\right.}{\partial x_{j}}-\frac{\partial \phi\left\langle\delta \bar{u}_{i} \delta \bar{u}_{j}\right\rangle_{f}}{\partial x_{j}} \\
& +\underbrace{\phi\left\langle\left(-\frac{\delta \bar{P}}{\rho} \delta_{i j}+v_{f} \frac{\partial \bar{u}_{i}}{\partial x_{j}}\right) n_{j} \delta_{\omega}\right\rangle_{f}}_{-\bar{F}_{\phi_{i}}} .
\end{aligned}
$$

In Eq. (14), $\bar{F}_{\phi}$ is the drag force applied by the fluid flow on the solid inclusions, $-\partial \phi\left\langle\delta \bar{u}_{i} \delta \bar{u}_{j}\right\rangle_{f} / \partial x_{j}$ and $-\partial \phi\left\langle\overline{u_{i}^{\prime} u_{j}^{\prime}}\right\rangle_{f} / \partial x_{j}$ respectively correspond to dispersion effects and macroscopic shear stresses. In pipe flows, it is usually modeled according to

$\bar{F}_{\phi_{i}}=f_{w} \frac{\left\langle\bar{u}_{i}\right\rangle_{f}\left\|\langle\bar{u}\rangle_{f}\right\|}{2 D_{h}}$

where $f_{w}$ is the friction factor. In order to analyze energy transfers between macroscopic and sub-filter scales, we define the three following kinetic energies:

averaged turbulence kinetic energy : $\langle\bar{k}\rangle_{f}=\frac{1}{2}\left\langle\overline{u_{i}^{\prime} u_{i}^{\prime}}\right\rangle_{f}$,

macroscale kinetic energy : $\quad \bar{E}^{M}=\frac{1}{2}\left\langle\bar{u}_{i}\right\rangle_{f}\left\langle\bar{u}_{i}\right\rangle_{f}$, 
dispersive kinetic energy : $\quad\left\langle\bar{E}^{m}\right\rangle_{f}=\frac{1}{2}\left\langle\delta \bar{u}_{i} \delta \bar{u}_{i}\right\rangle_{f}$.

Let us notice that there is no consensus in the research community as to which energies shall be considered [15,21]. Nevertheless, balance equations of the proposed three energies may be derived in a rigourous manner [18]. The balance equation of averaged turbulence kinetic energy is obtained by spatially averaging equation (9):

$$
\begin{aligned}
\phi \frac{\partial\langle\bar{k}\rangle_{f}}{\partial t}+\frac{\partial}{\partial x_{i}} \phi\left\langle\bar{u}_{i}\right\rangle_{f}\langle\bar{k}\rangle_{f} & =\underbrace{-\frac{\partial}{\partial x_{i}} \phi\left\langle\delta \bar{k} \delta \bar{u}_{i}\right\rangle_{f}}_{I} \underbrace{-\frac{\partial}{\partial x_{i}} \phi\left\langle\overline{\left.\left(\frac{P^{\prime}}{\rho}+k^{\prime}\right) u_{i}^{\prime}\right\rangle_{f}}\right.}_{I I} \\
& \underbrace{+\frac{\partial}{\partial x_{i}}\left(v_{f} \frac{\partial \phi\langle\bar{k}\rangle_{f}}{\partial x_{i}}\right)}_{I I I} \underbrace{-\phi\left\langle\bar{R}_{i j}\right\rangle_{f} \frac{\partial \phi\left\langle\bar{u}_{i}\right\rangle_{f}}{\partial x_{j}}}_{I V} \\
& \underbrace{-\phi\left\langle\delta \bar{R}_{i j} \frac{\partial \delta \bar{u}_{i}}{\partial x_{j}}\right\rangle_{f}}_{V} \underbrace{-\phi\langle\bar{\varepsilon}\rangle_{f}}_{V I},
\end{aligned}
$$

where

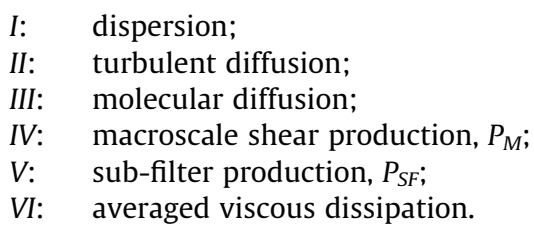

Subtracting Eq. (14) from Eq. (8), one can write a balance equation for the velocity spatial deviations:

$$
\begin{aligned}
& \phi \frac{\partial \delta \bar{u}_{i}}{\partial t}+\frac{\partial}{\partial x_{j}}\left(\phi\left\langle\bar{u}_{i}\right\rangle_{f} \delta \bar{u}_{j}\right)+\frac{\partial}{\partial x_{j}} \phi\left(\delta \bar{u}_{i} \bar{u}_{j}\right) \\
& =-\frac{1}{\rho} \frac{\partial \phi \delta \bar{P}}{\partial x_{i}}+\frac{\partial}{\partial x_{j}}\left(v_{f} \frac{\partial \phi \delta \bar{u}_{i}}{\partial x_{j}}\right)-\frac{\partial \phi \delta \bar{R}_{i j}}{\partial x_{j}}+\frac{\partial}{\partial x_{j}} \phi\left\langle\delta \bar{u}_{i} \delta \bar{u}_{j}\right\rangle_{f}+\bar{F}_{\phi_{i}} .
\end{aligned}
$$

The balance equation for $\bar{E}^{m}$ (dispervice kinetic energy) is then obtained by multiplying Eq. (20) by $\delta \bar{u}_{i}$ and by applying the spatial average. This equation reads

$$
\begin{aligned}
& \phi \frac{\partial\left\langle\bar{E}^{m}\right\rangle_{f}}{\partial t}+\frac{\partial}{\partial x_{i}} \phi\left\langle\bar{u}_{i}\right\rangle_{f}\left\langle\bar{E}^{m}\right\rangle_{f}=\underbrace{-\frac{1}{\rho} \frac{\partial \phi\left\langle\delta \bar{P} \delta \bar{u}_{i}\right\rangle_{f}}{\partial x_{i}}}_{I} \underbrace{+\frac{\partial}{\partial x_{i}}\left(v_{f} \frac{\partial \phi\left\langle\bar{E}^{m}\right\rangle_{f}}{\partial x_{i}}\right)}_{I I} \\
& \underbrace{-\phi\left\langle v_{f} \frac{\partial \delta \bar{u}_{i}}{\partial x_{j}} \frac{\partial \delta \bar{u}_{i}}{\partial x_{j}}\right\rangle_{f}}_{I V} \underbrace{-\frac{\partial}{\partial x_{j}} \phi\left\langle\delta \bar{R}_{i j} \delta \bar{u}_{i}\right\rangle_{f}}_{I I I} \\
& \underbrace{+\phi\left\langle\delta \bar{R}_{i j} \frac{\partial \delta \bar{u}_{i}}{\partial x_{j}}\right\rangle_{f}}_{V} \underbrace{+\phi\left\langle\bar{u}_{i}\right\rangle_{f} \bar{F}_{\phi_{i}}}_{V I} \\
& \underbrace{-\phi\left\langle\delta \bar{u}_{i} \delta \bar{u}_{j}\right\rangle_{f} \frac{\partial \phi\left\langle\bar{u}_{i}\right\rangle_{f}}{\partial x_{j}}}_{\text {VII }} \underbrace{-\frac{\partial}{\partial x_{i}} \phi\left\langle\delta \bar{E}^{m} \delta \bar{u}_{i}\right\rangle_{f}}_{\text {VIII }},
\end{aligned}
$$

where

I: pressure-velocity correlation;

II: diffusion;

III: $\quad$ wake dissipation, $\left\langle\bar{\varepsilon}_{w}\right\rangle_{f}$;

$I V$ : turbulent diffusion;

$V: \quad$ opposite of sub-filter production, $-P_{S F}$.

VI: drag;

VII: transfer from macroscale mean motion;

VIII: dispersion.
Finally, the balance equation for $\bar{E}^{M}$ is obtained by multiplying Eq. (14) by $\left\langle\bar{u}_{i}\right\rangle_{f}$ :

$$
\begin{aligned}
\phi \frac{\partial \bar{E}^{M}}{\partial t}+\frac{\partial}{\partial x_{i}} \phi\left\langle\bar{u}_{i}\right\rangle_{f} \bar{E}^{M} & =\underbrace{-\frac{1}{\rho} \frac{\partial}{\partial x_{i}}\left(\phi\langle\bar{P}\rangle_{f}\left\langle\bar{u}_{i}\right\rangle_{f}\right)}_{I} \underbrace{+\frac{\partial}{\partial x_{i}}\left(v_{f} \frac{\partial \phi \bar{E}^{M}}{\partial x_{i}}\right)}_{I I} \\
& \underbrace{-v_{f} \frac{\partial \phi\left\langle\bar{u}_{i}\right\rangle_{f}}{\partial x_{j}} \frac{\partial \phi\left\langle\bar{u}_{i}\right\rangle_{f}}{\partial x_{j}}}_{V} \underbrace{-\frac{\partial}{\partial x_{j}} \phi\left\langle\bar{u}_{i}\right\rangle_{f}\left\langle\bar{R}_{i j}\right\rangle_{f}}_{I V} \\
& \underbrace{+\phi\left\langle\delta \bar{R}_{i j}\right\rangle_{f} \frac{\partial \phi\left\langle\bar{u}_{i}\right\rangle_{f}}{\partial x_{j}}}_{V I I} \underbrace{-\frac{\partial}{\partial x_{j}} \phi\left\langle\bar{u}_{i}\right\rangle_{f}\left\langle\delta \bar{u}_{j}\right\rangle_{f} \frac{\left.\partial \phi \bar{u}_{j}\right\rangle_{f}}{\partial x_{j}}}_{V I} \underbrace{-\phi\left\langle\bar{u}_{i}\right\rangle_{f} \bar{F}_{\phi_{i}},}_{V I I I}
\end{aligned}
$$

where

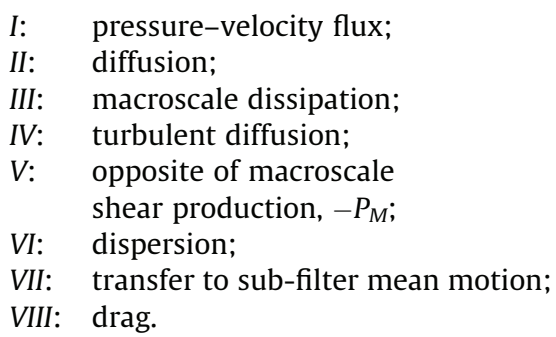

Let us notice that this latter equation is fully closed as soon as all contributions of the momentum equation (14) are closed. In other words, this equation is redundant with the momentum equation and is only used in order to highlight energy transfers.

Dispersive kinetic energy $\left\langle\bar{E}^{m}\right\rangle_{f}$ is mainly fed by the macroscale mean kinetic energy $\bar{E}^{M}$ through the drag force applied by the fluid flow on the solid inclusions. Since velocity gradients at macroscale are weak, the transfer between $\bar{E}^{M}$ and $\langle\bar{k}\rangle_{f}$ is negligible. At sub-filter scale, energy is mainly transferred form $\left\langle\bar{E}^{m}\right\rangle_{f}$ to $\langle\bar{k}\rangle_{f}$ through the so called sub-filter production $P_{S F}$. This contribution represents the interaction between turbulence and velocity gradients at the pore scale, say in our case at the channel scale. Considering the limit of a steady homogeneous flows with no macroscopic shear, Eq. (21) indicates that this sink term is a viscous dissipation induced by the velocity spatial deviation gradients, hereafter called wake dissipation and denoted $\left\langle\bar{\varepsilon}_{w}\right\rangle_{f}$. Let us notice that for laminar flows, this dissipation is not zero and is strictly equal to the work performed by the mean macroscopic flow against the drag force. Turbulent energy is finally dissipated into heat by way of viscous dissipation. Energy transfers between scales are summarized in Fig. 3.

\section{Derivation of a turbulence model based on the turbulent and dispersive kinetic energies}

The analysis of reference results (spatially averaged fine scale simulation results) have shown us that, in pipe flows, $\langle\bar{\varepsilon}\rangle_{f}$ never departs sustainably from equilibrium. Indeed, $\langle\bar{\varepsilon}\rangle_{f}$ can be modeled thanks to an algebraic closure relationship and we decide to drop its transport equation. On the contrary, $\left\langle\bar{E}^{m}\right\rangle_{f}$ encounters large and sustainable perturbations on a wide region of the flow. This statement questions the closure (4) proposed for the sub-filter production by Pinson et al. [18]. Furthermore, at the sub-filter scale, it is the exchange between $\left\langle\bar{E}^{m}\right\rangle_{f}$ and $\langle\bar{k}\rangle_{f}$ (through $P_{S F}$ ) that governs turbulent kinetic energy dynamic. This statement applies particularly at the boundary between porous and free flow regions where 


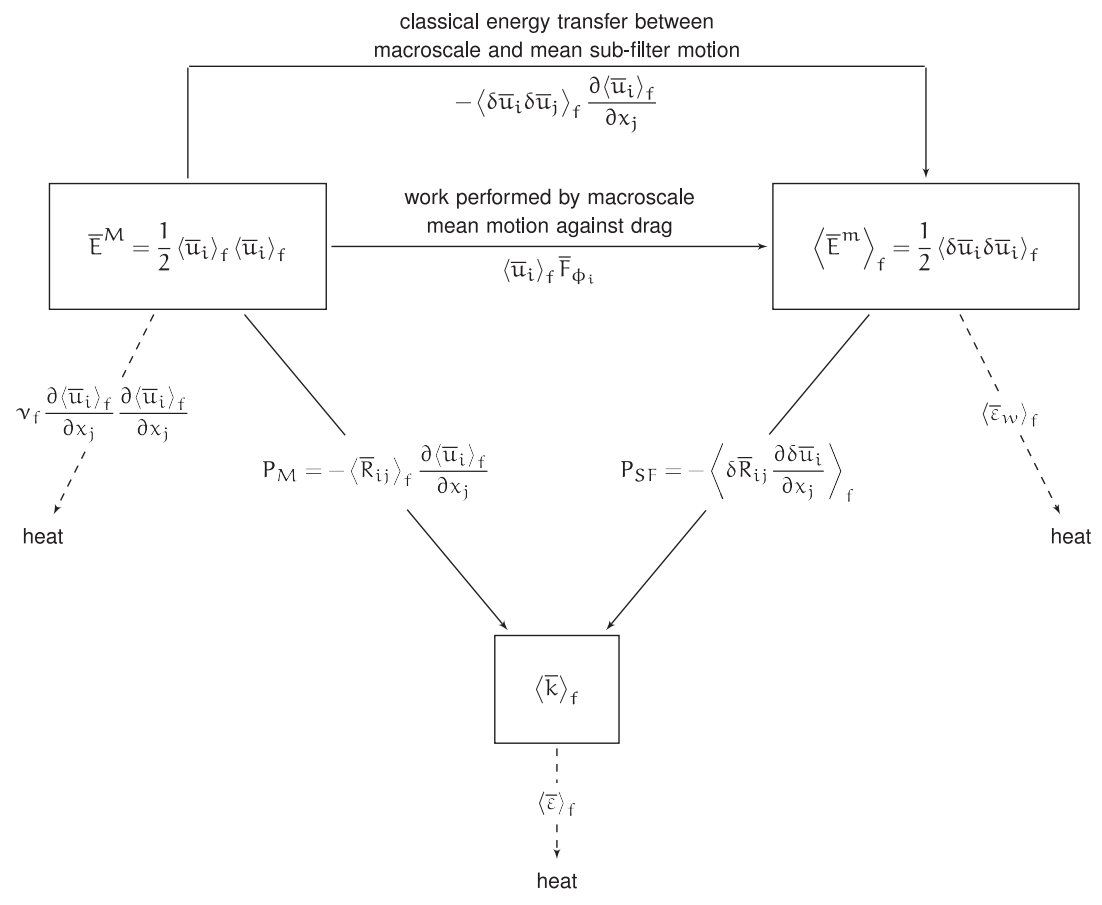

Fig. 3. Description of energy transfer for turbulent flows in porous media.

turbulent kinetic energy is only supplied with sub-filter production resulting from the vanishing of $\left\langle\bar{E}^{m}\right\rangle_{f}$ (see [2, Chapter 8]). Pinson et al. [18] introduced the idea that dispersive energy is representative of the degree of establishment of the flow and is tighly linked to dispersion. Since one of our major concern is to connect turbulence and dispersion [5] modeling, we choose to focus on the dispersive kinetic energy $\left\langle\bar{E}^{m}\right\rangle_{f}$ modeling. In this section, we follow those prescriptions and present a model based on Eqs. (19) and (21) completed, as in [18], by a postulated transport equation for wake dissipation $\left(\left\langle\bar{\varepsilon}_{w}\right\rangle_{f}\right)$.

\subsection{Macroscopic shear production}

Macroscopic Reynolds tensor $\left\langle\bar{R}_{i j}\right\rangle_{f}$ is usually modeled by Nakayama and Kuwahara [12], Pedras and de Lemos [16] and Pinson et al. [18]:

$-\phi\left\langle\bar{R}_{i j}\right\rangle_{f}=v_{t \phi}\left(\frac{\partial \phi\left\langle\bar{u}_{i}\right\rangle_{f}}{\partial x_{j}}+\frac{\partial \phi\left\langle\bar{u}_{j}\right\rangle_{f}}{\partial x_{i}}\right)-\frac{2}{3} \phi\langle\bar{k}\rangle_{f} \delta_{i j}$,

which is similar to the eddy-viscosity model for microscopic flows. Let us emphasize that the macroscopic turbulent diffusion coefficient $v_{t \phi}$ is not the spatial average of the local eddy viscosity $v_{t}$. It is given by $v_{t \phi}=C_{\mu}^{\prime}\langle\bar{k}\rangle_{f}^{2} /\langle\bar{\varepsilon}\rangle_{f}$, with $C_{\mu}=0.09$ [1,16,12]. Pinson [17] has shown that this hypothesis is questionable. However, this term vanishes in the cases studied below and thus will not be further investigated in this paper.

\subsection{Diffusion and dispersion}

Turbulent diffusion in Eq. (19) is usually modeled in the literature $[1,7,12,16,18]$ by a gradient approximation

$-\frac{\partial}{\partial x_{i}} \phi \overline{\left.\left(\frac{P^{\prime}}{\rho}+k^{\prime}\right) u_{i}^{\prime}\right\rangle_{f}}=\frac{\partial}{\partial x_{i}}\left(\frac{v_{t \phi}}{\sigma_{k}} \frac{\partial \phi\langle\bar{k}\rangle_{f}}{\partial x_{i}}\right)$.

where $\sigma_{k}=1$. Dispersion effects in (19) can be split into a contribution due to velocity heterogeneities, hereafter called passive dispersion, and a contribution related to sub-filter production and viscous dissipation [17], called active dispersion. Passive dispersion can easily be modeled using the thermal dispersion model of Drouin et al. [5] by introducing Lewis numbers [12]. In this paper, we choose to neglect active dispersion and to model dispersion of macroscopic turbulent kinetic energy according to

$-\frac{\partial}{\partial x_{i}} \phi\left\langle\delta \bar{k} \delta \bar{u}_{i}\right\rangle_{f}=\frac{\partial}{\partial x_{i}}\left(\frac{\mathcal{D}_{i j}^{P}}{L e_{E}} \frac{\partial \phi\langle\bar{k}\rangle_{f}}{\partial x_{j}}\right)$,

where $\mathcal{D}_{i j}^{p}$ is the passive thermal dispersion tensor. Within the same frame, pressure-velocity correlation, dispersion and turbulent diffusion in (21) are modeled collectively as

$$
\begin{gathered}
-\frac{1}{\rho} \frac{\partial \phi\left\langle\delta \bar{P} \delta \bar{u}_{i}\right\rangle_{f}}{\partial x_{i}}-\frac{\partial}{\partial x_{i}} \phi\left\langle\delta \bar{E}^{m} \delta \bar{u}_{i}\right\rangle_{f}-\frac{\partial}{\partial x_{i}} \phi\left\langle\delta \bar{R}_{i j} \delta \bar{u}_{i}\right\rangle_{f} \\
=\frac{\partial}{\partial x_{i}}\left(\frac{v_{t \phi}}{\sigma_{E}} \frac{\partial \phi\left\langle\bar{E}^{m}\right\rangle_{f}}{\partial x_{i}}\right)+\frac{\partial}{\partial x_{i}}\left(\frac{\mathcal{D}_{i j}^{P}}{L e_{E}} \frac{\partial \phi\left\langle\bar{E}^{m}\right\rangle_{f}}{\partial x_{j}}\right) .
\end{gathered}
$$

The Lewis numbers for mechanical dispersion $L e_{k}$ and $L e_{E}$ are taken equal to 1 [13]. In stratified media, we propose two possibilities for $\mathcal{D}_{z z}^{P}$ modeling:

1. the straightforward use of Drouin et al. [5] model,

2. to introduce a dispersion coefficient based upon quantities solved by the turbulence model.

Actually, if one considers the following proposition for the dynamical dispersion coefficient

$\mathcal{D}_{z z}^{P}=\frac{v_{d \phi}}{\operatorname{Pr}_{d}}=\frac{C_{\mu}^{\prime \prime}}{\operatorname{Pr}_{d}} \frac{\left\langle\bar{E}^{m}\right\rangle_{f}^{2}}{\left\langle\bar{\varepsilon}_{w}\right\rangle_{f}}$,

one can easily show that it is possible to recover the dispersion model proposed in Drouin et al. [5] when flows are fully developed (see Section 4.1 hereafter). Comparison between Eq. (27) and the model of Drouin et al. [5] for turbulent flows in pipes leads to

$\frac{C_{\mu}^{\prime \prime}}{P r_{d}}=5.85$ 
For several Prandtl numbers, comparisons between the dynamical dispersion model, given by (27), and the model of Drouin et al. [5] are presented in Fig. 4. One can see that both formulations are equivalent for fully developed flows. Due to its simplicity, and because it is an elegant way to bridge the gap between dispersion modeling and macroscale turbulence modeling, we choose here to use the dynamical dispersion model (27).

\subsection{Dissipation rate and sub-filter production}

Given the fact that sub-filter production represents an energy transfer between sub-filter mean motion and turbulent kinetic energies, it is modeled by

$\frac{P_{S F}}{P_{S F \infty}}=\left(\frac{\langle\bar{k}\rangle_{f}}{\langle\bar{k}\rangle_{f, \infty}}\right)^{a}\left(\frac{\left\langle\bar{E}^{m}\right\rangle_{f}}{\left\langle\bar{E}^{m}\right\rangle_{f, \infty}}\right)^{b}$

where $a$ and $b$ are constants and $P_{S F \infty},\langle\bar{k}\rangle_{f, \infty}$ and $\left\langle\bar{E}^{m}\right\rangle_{f, \infty}$ are representative values of turbulent kinetic energy and dispersive kinetic energy for the flow under consideration.

Spatially averaged viscous dissipation represents the part of macroscopic turbulent kinetic energy dissipated into heat. It is therefore modeled, by analogy with local $\bar{k}-\ell$ turbulence models as

$\frac{\langle\bar{\varepsilon}\rangle_{f}}{\langle\bar{\varepsilon}\rangle_{f, \infty}}=\left(\frac{\langle\bar{k}\rangle_{f}}{\langle\bar{k}\rangle_{f, \infty}}\right)^{n}$,

where $n$ is a constant and $\langle\bar{k}\rangle_{f, \infty}$ and $\langle\bar{\varepsilon}\rangle_{f, \infty}$ are representative values of turbulent kinetic energy dissipation for the flow under consideration.

\subsection{Wake dissipation}

Wake dissipation is induced by the velocity deviation gradients and is defined by:

$\left\langle\bar{\varepsilon}_{w}\right\rangle_{f}=\left\langle v_{f} \frac{\partial \delta \bar{u}_{i}}{\partial x_{j}} \frac{\partial \delta \bar{u}_{i}}{\partial x_{j}}\right\rangle_{f}$.

In a media with solid structures embedded, it is controlled by wall boundary layers. One can then presume that evolution of wake dissipation results from a competition between production due to the friction with the wall (represented by the drag) and dissipation by viscous effects. Following Pinson et al. [18], the $\left\langle\bar{\varepsilon}_{w}\right\rangle_{f}$ transport equation is postulated:

$$
\begin{aligned}
\phi \frac{\partial\left\langle\bar{\varepsilon}_{w}\right\rangle_{f}}{\partial t}+\frac{\partial \phi\left\langle\bar{u}_{i}\right\rangle_{f}\left\langle\bar{\varepsilon}_{w}\right\rangle_{f}}{\partial x_{i}}= & \frac{\partial}{\partial x_{i}}\left[\left(v_{f}+\frac{v_{t \phi}}{\sigma_{\epsilon_{w}}}\right) \frac{\partial \phi\left\langle\bar{\varepsilon}_{w}\right\rangle_{f}}{\partial x_{i}}\right] \\
& +\frac{\partial}{\partial x_{i}}\left(\frac{\mathcal{D}_{i j}^{P}}{L e_{w}} \frac{\partial \phi\left\langle\bar{\varepsilon}_{w}\right\rangle_{f}}{\partial x_{j}}\right) \\
& +\frac{C_{\varepsilon_{w, 1}}}{\tau_{w_{1}}} \phi\left\langle\bar{u}_{i}\right\rangle_{f} \bar{F}_{\phi_{i}}-\frac{C_{\varepsilon_{w, 2}}}{\tau_{w_{2}}} \phi\left\langle\bar{\varepsilon}_{w}\right\rangle_{f},
\end{aligned}
$$

where $\tau_{w_{1}}$ and $\tau_{w_{2}}$ are characteristic time scales and $C_{\varepsilon_{w 1}}$ and $C_{\varepsilon_{w 2}}$ are constants. By analogy with local $\bar{k}-\bar{\varepsilon}$ models, we choose $\tau_{w_{1}}=\tau_{w_{2}}=\left\langle\bar{E}^{m}\right\rangle_{f} /\left\langle\bar{\varepsilon}_{w}\right\rangle_{f}$. Lewis number $L e_{w}$ is taken equal to one.

\subsection{Friction factor}

Wake dissipation and friction are two macroscopic effects induced by the presence of obstacles and walls. Based on a dimensional argument, we assume $\left\langle\bar{\varepsilon}_{w}\right\rangle_{f} \propto u_{\tau}^{3} / D_{h} \propto\left\langle\bar{u}_{z}\right\rangle_{f}^{3} f_{w}^{3 / 2} / D_{h}$. We thus propose

$\frac{f_{w}}{f_{w \infty}}=\left(\frac{\left\langle\bar{\varepsilon}_{w}\right\rangle_{f}}{\left\langle\bar{\varepsilon}_{w}\right\rangle_{f, \infty}}\right)^{2 / 3}$,

where $f_{w \infty}$ and $\left\langle\bar{\varepsilon}_{w}\right\rangle_{f, \infty}$ are representative values of the friction factor and wake dissipation for the flow under consideration. For instance, for flows in channels, representative values correspond to fully developed flow limit. This closure is simpler than the one proposed by Pinson et al. [18].

\section{Calibration methodology for the model constants: the stratified porous media configuration}

In this section, we propose a general methodology for the calibration of our macroscopic turbulence model for stratified porous media with constant porosity. We apply the calibration procedure for media composed of flat plates or circular pipes (Fig. 5) oriented in the $z$ direction. Reynolds number is given by $R e=\left\langle\bar{u}_{z}\right\rangle_{f} D_{h} / v_{f}$. In such media, no eddy larger than the pore size can exist $[17,7]$. There is no macroscopic velocity gradient and the macroscopic shear production vanishes. In the configurations under study, Eqs. (19), (21) and (32) can be simplified:

$$
\begin{aligned}
\frac{D\langle\bar{k}\rangle_{f}}{D t}= & \frac{\partial}{\partial z}\left[\left(v_{f}+\frac{v_{t \phi}}{\sigma_{k}}+\mathcal{D}_{z z}^{P}\right) \frac{\partial\langle\bar{k}\rangle_{f}}{\partial z}\right] \\
& +P_{S F \infty}\left(\frac{\langle\bar{k}\rangle_{f}}{\langle\bar{k}\rangle_{f, \infty}}\right)^{a}\left(\frac{\left\langle\bar{E}^{m}\right\rangle_{f}}{\left\langle\overline{E^{m}}\right\rangle_{f, \infty}}\right)^{b}-\langle\bar{\varepsilon}\rangle_{f, \infty}\left(\frac{\langle\bar{k}\rangle_{f}}{\langle\bar{k}\rangle_{f, \infty}}\right)^{n},
\end{aligned}
$$

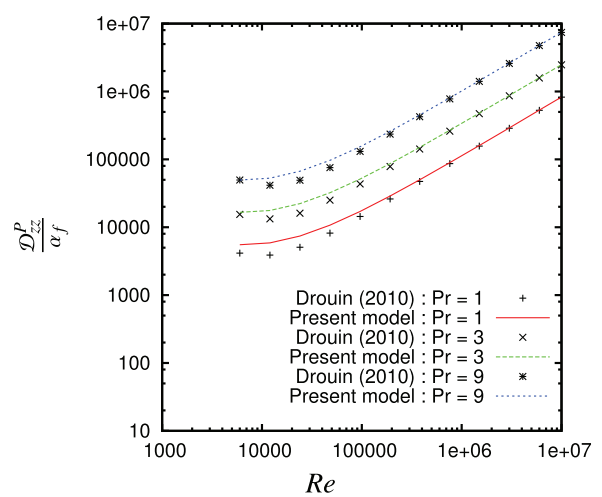

(b) Circular pipe

Fig. 4. Comparison between the present model and the model of Drouin et al. [5] for fully developed turbulent flows in pipes. 


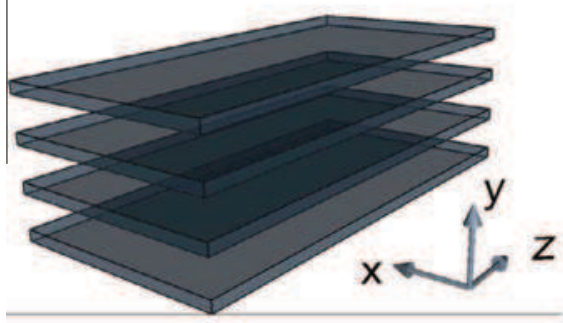

(a) Flat plates

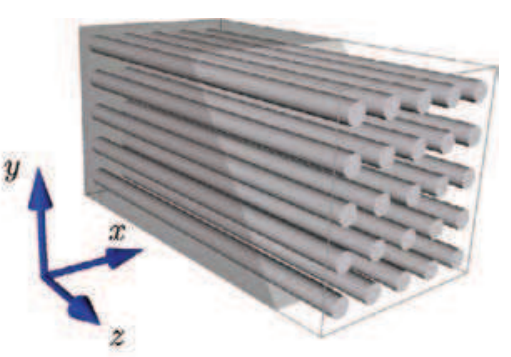

(b) Circular pipes

Fig. 5. Example of stratified porous medium: description of a porous medium composed of flat plates or circular pipes.

$$
\begin{aligned}
\frac{D\left\langle\bar{E}^{m}\right\rangle_{f}}{D t}= & \frac{\partial}{\partial z}\left[\left(v_{f}+\frac{v_{t \phi}}{\sigma_{E}}+\mathcal{D}_{z z}^{P}\right) \frac{\partial\left\langle\bar{E}^{m}\right\rangle_{f}}{\partial z}\right]+\left\langle\bar{u}_{z}\right\rangle_{f} \bar{F}_{\phi_{z}} \\
& -P_{S F \infty}\left(\frac{\langle\bar{k}\rangle_{f}}{\langle\bar{k}\rangle_{f, \infty}}\right)^{a}\left(\frac{\left\langle\bar{E}^{m}\right\rangle_{f}}{\left\langle\bar{E}^{m}\right\rangle_{f, \infty}}\right)^{b}-\left\langle\bar{\varepsilon}_{w}\right\rangle_{f}, \\
\frac{D\left\langle\bar{\varepsilon}_{w}\right\rangle_{f}}{D t}= & \frac{\partial}{\partial z}\left[\left(v_{f}+\frac{v_{t \phi}}{\sigma_{\epsilon_{w}}}+\mathcal{D}_{z z}^{P}\right) \frac{\partial\left\langle\bar{\varepsilon}_{w}\right\rangle_{f}}{\partial z}\right]+C_{\varepsilon_{w, 1}} \frac{\left\langle\bar{\varepsilon}_{w}\right\rangle_{f}}{\left\langle\bar{E}^{m}\right\rangle_{f}}\left\langle\bar{u}_{z\rangle_{f}} \bar{F}_{\phi_{z}}\right. \\
& -C_{\varepsilon_{w, 2}} \frac{\left\langle\bar{\varepsilon}_{w}\right\rangle_{f}^{2}}{\left\langle\bar{E}^{m}\right\rangle_{f}}
\end{aligned}
$$

Let us recall that the macroscopic turbulence model shall be able to

1. reach the fully developed flow limit,

2. provide a good dynamical response to perturbations.

Priority is given to asymptotic behavior prediction since, for most devices under study (heat exchangers, reactor cores, ...), the hydraulic diameter is much smaller than the typical length of the device and the length of establishment is much smaller than the length of the device. One could say that the transition between free flow and porous media induces a "large perturbation". This is why we shall then focus on the capability of the model to predict the dynamical establishment of quantities in the inlet region. The following calibration method aims to satisfy those two constraints.

\subsection{Asymptotic state modeling}

Far downstream the inlet, all profiles reach non-evolving levels and shapes. This asymptotic state, denoted ". ${ }_{\infty}$ ", corresponds to the fully developed flow limit. It depends upon geometry and, within turbulent regime, on the Reynolds number of the flow. When deriving macroscopic turbulence model, one shall consider the capability of the model to asymptotically reach the fully developed flow limit as a major objective.

The friction factor asymptotic value $f_{w_{\infty}}$ may be given by the correlations available in the literature [10]. For turbulent flows in pipes, established friction velocity is defined by $u_{\tau, \infty}=\left\langle\bar{u}_{z}\right\rangle_{f} \sqrt{f_{w \infty} / 8}$. Wake dissipation and friction have the same origin. Consistently with relation (33), we propose:

$\left\langle\bar{\varepsilon}_{w}\right\rangle_{f, \infty} \propto \frac{u_{\tau, \infty}^{3}}{D_{h}}=C_{w}(R e) \frac{\left\langle\bar{u}_{z}\right\rangle_{f}^{3}}{2 D_{h}} f_{w \infty}^{3 / 2}$.

Using reference velocity profiles for fully developed flows in pipes, we show that $C_{w}(R e)$ can be assumed constant and equal to 3. Following the same approach, we assume that the asymptotic value of turbulent kinetic energy is proportional to $u_{\tau, \infty}^{2}$

$\langle\bar{k}\rangle_{f, \infty}=c_{k} u_{\tau, \infty}^{2}=c_{k}\left\langle\bar{u}_{z}\right\rangle_{f}^{2} \frac{f_{w \infty}}{8}$,
Comparisons with results obtained for fully developed flows with low-Reynolds $\bar{k}-\bar{\varepsilon}$ fine scale simulations lead to

$c_{k}=1.82$ for plane channels,

$c_{k}=2.32$ for circular pipes.

Considering the equilibrium of kinetic turbulent energy and dispersive energy when the flow is developed ((34) and (35)), one can write

$$
\begin{aligned}
P_{S F \infty} & =\langle\bar{\varepsilon}\rangle_{f, \infty}, \\
P_{S F \infty} & =\left\langle\bar{u}_{z}\right\rangle_{f} \bar{F}_{\phi_{z}}^{\infty}-\left\langle\bar{\varepsilon}_{w}\right\rangle_{f, \infty} .
\end{aligned}
$$

Using (15), we get

$P_{S F \infty}=\langle\bar{\varepsilon}\rangle_{f, \infty}=\frac{\left\langle\bar{u}_{z}\right\rangle_{f}^{3}}{2 D_{h}} f_{w \infty}\left(1-C_{w} f_{w \infty}^{1 / 2}\right)$.

Finally, the equilibrium of wake dissipation balance equation (36) imposes:

$C_{\varepsilon_{w, 2}}=C_{\varepsilon_{w, 1}} \frac{\left\langle\bar{u}_{z}\right\rangle_{f} \bar{F}_{\phi_{z}}^{\infty}}{\left\langle\bar{\varepsilon}_{w}\right\rangle_{f, \infty}}$

For quasi-parallel flows, $\left\langle\bar{E}^{m}\right\rangle_{f} \simeq\left\langle\delta \bar{u}_{z} \delta \bar{u}_{z}\right\rangle_{f} / 2$ and thus has the structure of a dispersion contribution. Consequently we inspire of the available thermal dispersion models to derive the asymptotic model for $\left\langle\bar{E}^{m}\right\rangle_{f, \infty}$. The following thermal dispersion model gives satisfactory results [5]:

$-\left\langle\delta \bar{u}_{z} \bar{T}_{f}\right\rangle_{f}=\mathcal{D}_{z z}^{P} \frac{\partial\left\langle\bar{T}_{f}\right\rangle_{f}}{\partial z}+\mathcal{D}_{z}^{A} \frac{\left\langle\bar{\Phi} \delta_{\omega}\right\rangle_{f}}{\left(\rho C_{p}\right)_{f}}$

where $\left\langle\bar{\Phi} \delta_{\omega}\right\rangle_{f} /\left(\rho C_{p}\right)_{f}$ is the source term due to the wall heat flux $\bar{\Phi}$ in $\left\langle\bar{T}_{f}\right\rangle_{f}$ balance equation. By analogy with (42), we propose

$-\left\langle\delta \bar{u}_{z} \delta \bar{u}_{z}\right\rangle_{f}=\mathcal{D}_{z z}^{P} \frac{\partial\left\langle\bar{u}_{z}\right\rangle_{f}}{\partial z}-\mathcal{D}_{z}^{A} \bar{F}_{\phi_{z}}$,

where $\mathcal{D}_{z z}^{P}$ and $\mathcal{D}_{z}^{A}$ are passive and active dispersion coefficients. Doing so, the analogy between heat exchange (source/ sink term for temperature) and friction (sink term for momentum) is clearly underlying. When porosity is constant, Eq. (43) further simplifies

$\left\langle\delta \bar{u}_{z} \delta \bar{u}_{z}\right\rangle_{f}=\mathcal{D}_{z}^{A} \bar{F}_{\phi_{z}}=\mathcal{D}_{z}^{A} \frac{\left\langle\bar{u}_{z}\right\rangle_{f}^{2}}{2 D_{h}} f_{w}=4 \mathcal{D}_{z}^{A^{*}} u_{\tau, \infty}^{2}$,

and we get:

$\left\langle\bar{E}^{m}\right\rangle_{f, \infty}=2 \mathcal{D}_{z}^{A^{*}} u_{\tau, \infty}^{2}$

We have shown in [5] that it is possible to approach the thermal active dispersion coefficient, $\mathcal{D}_{z}^{A^{*}}$, by simple functions depending on $\mathrm{Pr}$ and $R e$ numbers. For the dispersive energy, we transpose this model 
by assuming $P r=1$ and, using Eqs. (45) and (47) of Drouin et al. [5], we find:

$\mathcal{D}_{z}^{A^{*}}=1.63+3692 /\left(\sqrt{f_{w_{\infty}}} \operatorname{Re}\right)$ for plane channels,

$\mathcal{D}_{z}^{A^{*}}=2.1+2623 /\left(\sqrt{f_{w \infty}} R e\right)$ for circular pipes.

We can summarize the calibration of asymptotic values in the following way:

1. Select a friction correlation and the $c_{k}$ parameter adapted to your geometry. This shall give you $f_{w \infty}$ and $u_{\tau, \infty}$.

2. Evaluate $\left\langle\bar{\varepsilon}_{w}\right\rangle_{f, \infty},\langle\bar{k}\rangle_{f, \infty}$ and $P_{S F \infty}$ thanks to respectively (37), (38) and (40).

3. Evaluate $\left\langle\bar{E}^{m}\right\rangle_{f, \infty}$ thanks to (45) and (46).

In Figs. 6 and 7, those correlations for asymptotic values are successfully compared with reference results given by spatially averaged fine scale simulations.

\subsection{Dynamical behavior prediction}

The dynamic behavior of the macroscopic model is determined by the values of the constants $a, b, n$ and $C_{\varepsilon_{w, 1}}$. In order to evaluate
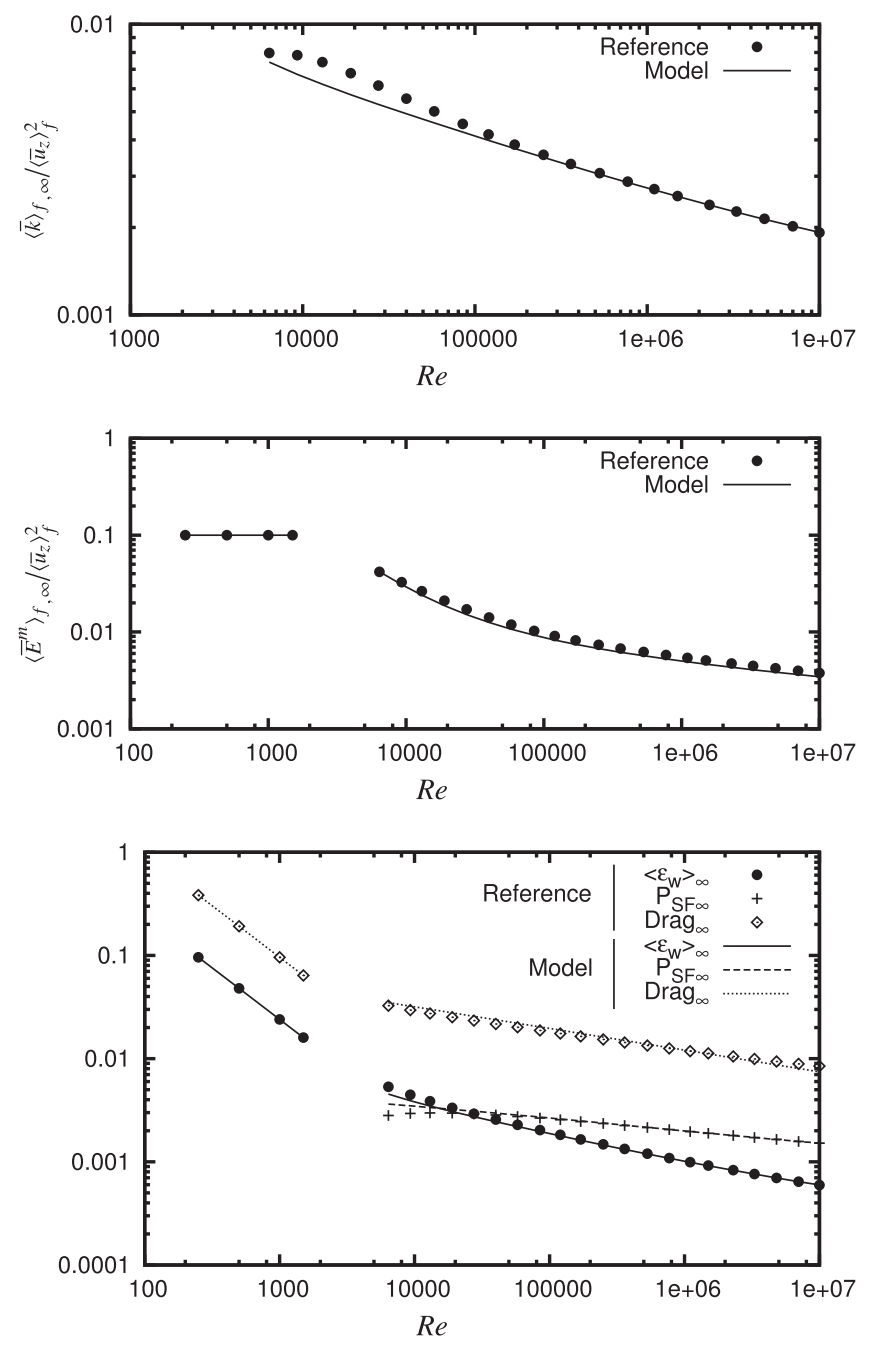

Fig. 6. Asymptotic states: evaluation of expressions (37), (40), (38) and (45) for flows in plane channels. The source terms $\left\langle\bar{\varepsilon}_{w}\right\rangle_{f \infty}, P_{S F \infty}$ and $\left\langle\bar{u}_{z}\right\rangle_{f} \bar{F}_{\phi_{z}}$ are adimensioned by $\left\langle\bar{u}_{z}\right\rangle_{f}^{3} / 2 D_{h}$.
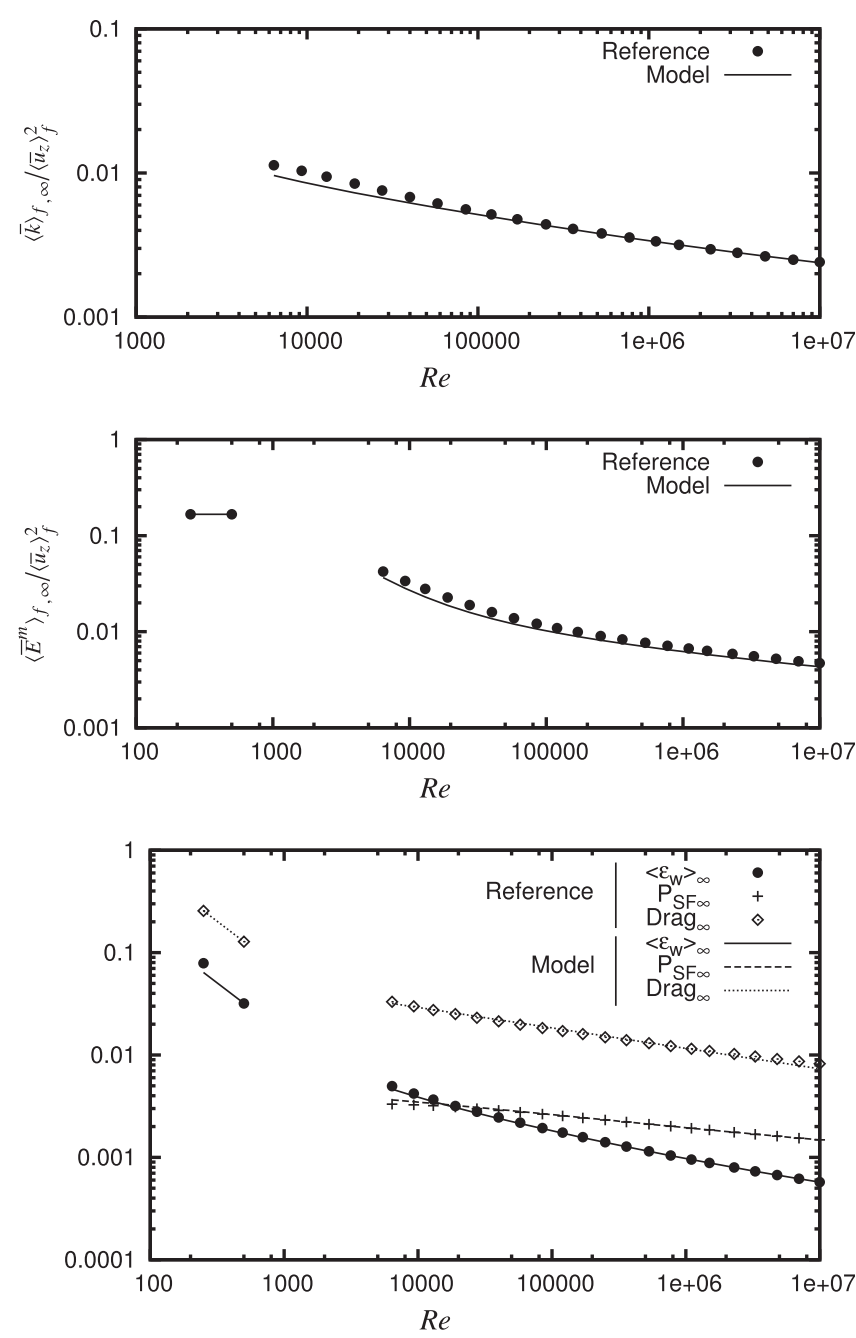

Fig. 7. Asymptotic states: evaluation of expressions (37), (40), (38) and (45) for flows in circular pipes. The source terms $\left\langle\bar{\varepsilon}_{w}\right\rangle_{f, \infty}, P_{S F_{\infty}}$ and $\left\langle\bar{u}_{z}\right\rangle_{f} \bar{F}_{\phi_{z}}^{\infty}$ are adimensioned by $\left\langle\bar{u}_{z}\right\rangle_{f}^{3} / 2 D_{h}$.

$a, b$ and $n$, a simplified system is considered. From the observation of spatially filtered fine scale simulations (see Fig. 8 for instance), we have deduced that dispersion and diffusion can be neglected in the simplified system and that turbulent variables may be split into two groups:

- the "rapid" group, that achieves its asymptotic state in less than five hydraulic diameters,

- the "slow" group, that need about $25 D_{h}$ in plane channel configuration, and $35 D_{h}$ in circular pipe configuration, to achieve its asymptotic state.

Drag and wake dissipation belong to the rapid group while the slow group is composed of sub-filter production, dispersive kinetic energy, turbulent kinetic energy and its dissipation rate. Furthermore, once the rapid group has reach its asymptotic state, the variables of the second group exhibit damped oscillations. Since we are mostly interested in those oscillations, drag and wake dissipation are assumed constantly equal to their asymptotic values in Eqs. (34) and (35). Consequently, for $z>5 D_{h}$, the $\langle\bar{k}\rangle_{f}-\left\langle\bar{E}^{m}\right\rangle_{f}$ system might be approached by the following damped oscillator system:

$\frac{d \mathbb{K}}{d t}=\frac{P_{S F \infty}}{\langle\bar{k}\rangle_{f, \infty}}\left(\mathbb{K}^{a} \mathbb{E}^{b}-\mathbb{K}^{n}\right)$, 


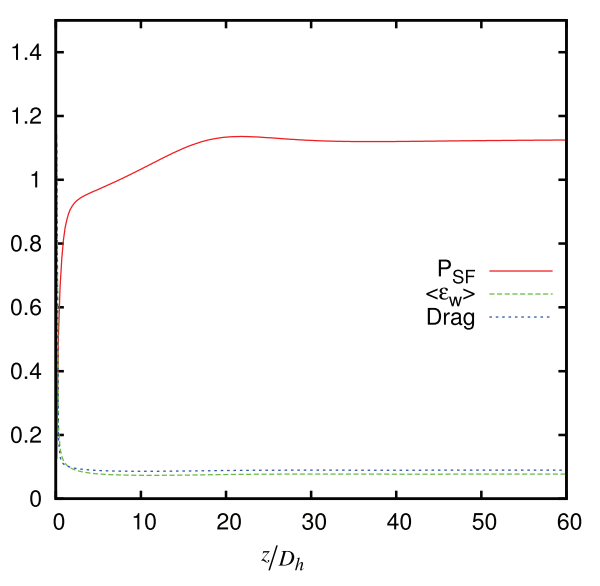

(a) Plane channel

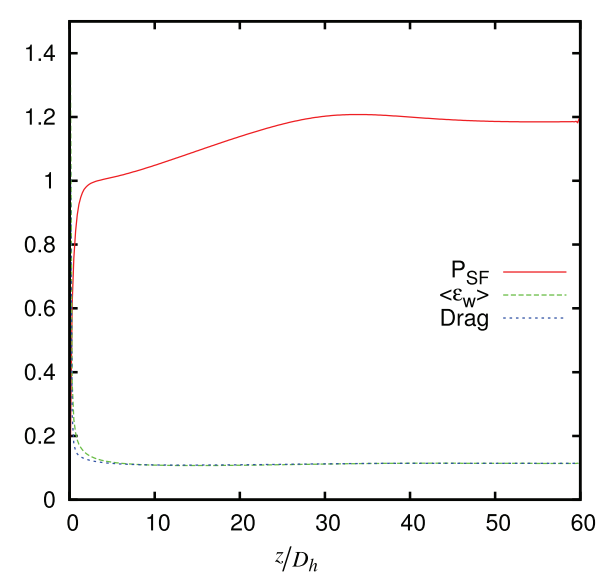

(b) Circular pipe

Fig. 8. Evolutions of sub-filter production, drag and wake dissipation for turbulent plane channel flows. Each quantity $\xi$ is adimensioned by $\left|\xi_{\text {inlet }}-\xi_{\infty}\right|$. The length of establishment associated to $P_{S F}$ is much larger than the one of the drag and wake dissipation.

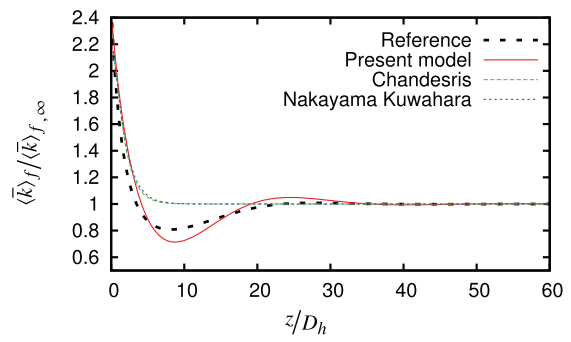

(a) Evolution of $\langle\bar{k}\rangle_{f} /\langle\bar{k}\rangle_{f, \infty}$

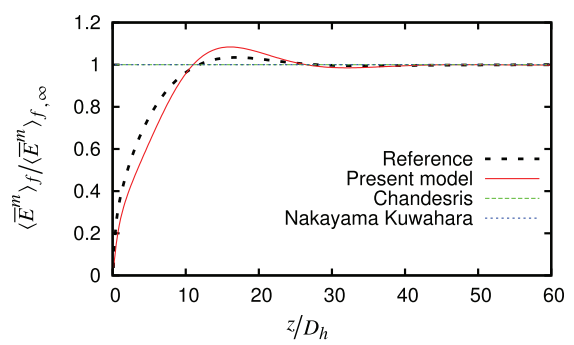

(c) Evolution of $\left\langle\bar{E}^{m}\right\rangle_{f} /\left\langle\bar{E}^{m}\right\rangle_{f, \infty}$

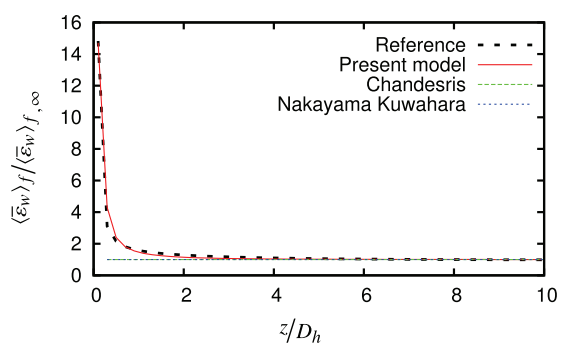

(e) Evolution of $\left\langle\bar{\varepsilon}_{w}\right\rangle_{f} /\left\langle\bar{\varepsilon}_{w}\right\rangle_{f, \infty}$

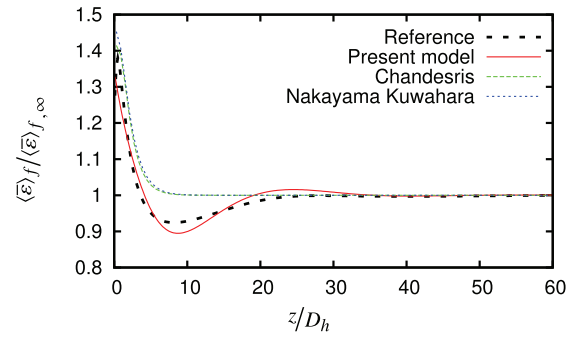

(b) Evolution of $\langle\bar{\varepsilon}\rangle_{f} /\langle\bar{\varepsilon}\rangle_{f, \infty}$



(d) Evolution of $\left(\left\langle\bar{E}^{m}\right\rangle_{f}+\langle\bar{k}\rangle_{f}\right) /\left(\left\langle\bar{E}^{m}\right\rangle_{f, \infty}+\langle\bar{k}\rangle_{f, \infty}\right)$

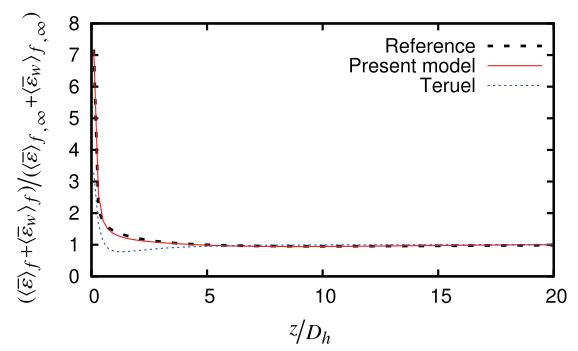

(f) Evolution of $\left(\langle\bar{\varepsilon}\rangle_{f}+\left\langle\bar{\varepsilon}_{w}\right\rangle_{f}\right) /\left(\langle\bar{\varepsilon}\rangle_{f}+\left\langle\bar{\varepsilon}_{w}\right\rangle_{f}\right)$

Fig. 9. Macroscopic turbulence model results for turbulent flow in stratified porous media composed of flat plates, $R e=7.6 \times 10^{4} ;\left\langle\bar{k}_{0}\right\rangle_{f} /\langle\bar{k}\rangle_{f, \infty}=4$. Comparison of the present model to reference results and to the models of Chandesris et al. [1], Nakayama and Kuwahara [13] and Teruel and Rizwan-uddin [22].

$\frac{d \mathbb{E}}{d t}=\frac{P_{S F \infty}}{\left\langle\bar{E}^{m}\right\rangle_{f, \infty}}\left(1-\mathbb{K}^{a} \mathbb{E}^{b}\right)$,

where $\mathbb{K}=\langle\bar{k}\rangle_{f} /\langle\bar{k}\rangle_{f, \infty}, \mathbb{E}=\left\langle\bar{E}^{m}\right\rangle_{f} /\left\langle\bar{E}^{m}\right\rangle_{f, \infty}$ and $t=z /\left\langle\bar{u}_{z}\right\rangle_{f}$.

The $\mathbb{K}-\mathbb{E}$ is then linearized, according to the following decomposition (see [4, p. 195]):

$\mathbb{E}=E_{0}+E_{1}$ and $\mathbb{K}_{0}=K_{0}+K_{1} \quad$ with $K_{0}=E_{0}=1$ and

$E_{1} \ll E_{0}, K_{1} \ll K_{0}$.
According to this decomposition into small perturbations, we approach, for any quantity $g$ and exponent $q$ :

$g^{q} \simeq g_{0}^{q}+q g_{0}^{q-1} g_{1}$.

System (46) and (47) thus may read:

$\frac{d K_{1}}{d t}=\frac{P_{S F \infty}}{\langle\bar{k}\rangle_{f, \infty}}\left[(a-n) K_{1}+b E_{1}\right]$ 


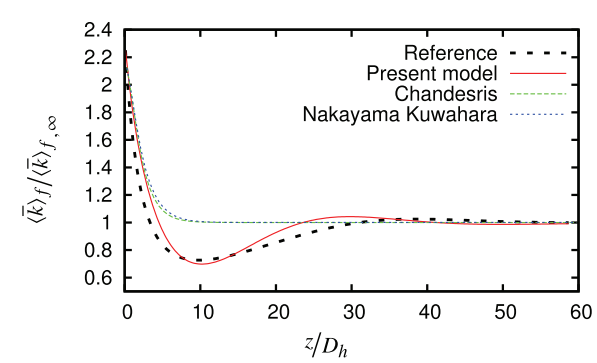

(a) Evolution of $\langle\bar{k}\rangle_{f} /\langle\bar{k}\rangle_{f, \infty}$



(c) Evolution of $\left\langle\bar{E}^{m}\right\rangle_{f} /\left\langle\bar{E}^{m}\right\rangle_{f, \infty}$

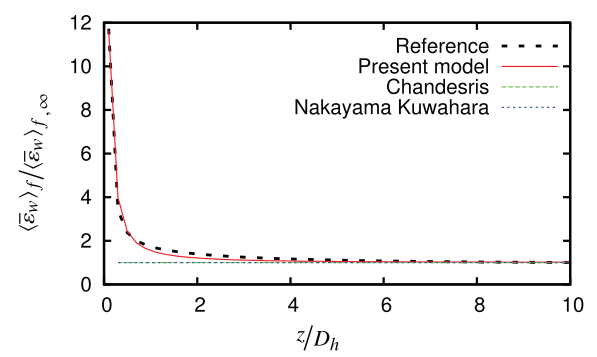

(e) Evolution of $\left\langle\bar{\varepsilon}_{w}\right\rangle_{f} /\left\langle\bar{\varepsilon}_{w}\right\rangle_{f, \infty}$

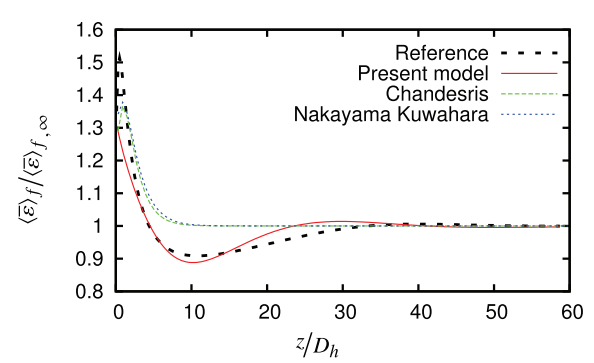

(b) Evolution of $\langle\bar{\varepsilon}\rangle_{f} /\langle\bar{\varepsilon}\rangle_{f, \infty}$

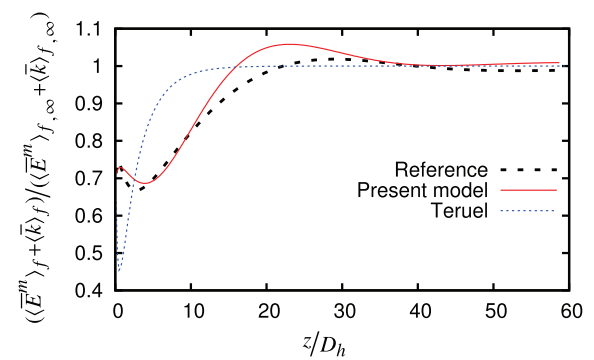

(d) Evolution of $\left(\left\langle\bar{E}^{m}\right\rangle_{f}+\langle\bar{k}\rangle_{f}\right) /\left(\left\langle\bar{E}^{m}\right\rangle_{f_{, \infty}}+\langle\bar{k}\rangle_{f, \infty}\right)$

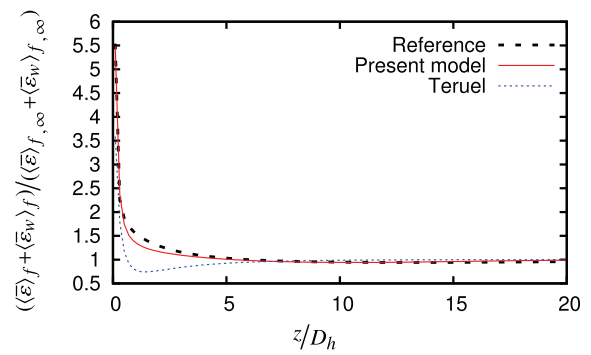

(f) Evolution of $\left(\langle\bar{\varepsilon}\rangle_{f}+\left\langle\bar{\varepsilon}_{w}\right\rangle_{f}\right) /\left(\langle\bar{\varepsilon}\rangle_{f, \infty}+\left\langle\bar{\varepsilon}_{w}\right\rangle_{f, \infty}\right)$

Fig. 10. Macroscopic turbulence model results for turbulent flow in stratified porous media composed of circular pipes, $R e=7.6 \times 10^{4} ;\left\langle\bar{k}_{0}\right\rangle_{f} /\langle\bar{k}\rangle_{f, \infty}=4$. Comparison of the present model to reference results and to the models of Chandesris et al. [1], Nakayama and Kuwahara[13] and Teruel and Rizwan-uddin [22].

$\frac{d E_{1}}{d t}=-\frac{P_{S F \infty}}{\left\langle\bar{E}^{m}\right\rangle_{f, \infty}}\left(a K_{1}+b E_{1}\right)$

The linearized system (50) and (51) can be analytically solved and, if one looks for damped oscillations behavior, its solutions are:

$\mathbb{K}(t)=1-\frac{\left\langle\bar{E}^{m}\right\rangle_{f, \infty}}{a} e^{-t / \tau} \mathcal{A}\left[\left(\frac{b}{\left\langle\bar{E}^{m}\right\rangle_{f, \infty}}+\frac{a-n}{\langle\bar{k}\rangle_{f, \infty}}\right) \cos \omega t-\frac{2 \omega}{P_{S F \infty}} \sin \omega t\right]$,

$\mathbb{E}(t)=1+\mathcal{A} e^{-t / \tau} \cos \omega t$,

where

$\frac{1}{\tau}=\frac{P_{S F \infty}}{2}\left(\frac{b}{\left\langle\bar{E}^{m}\right\rangle_{f, \infty}}-\frac{a-n}{\langle\bar{k}\rangle_{f, \infty}}\right)$

$\omega=\frac{P_{S F \infty}}{2}\left[\frac{4 b n}{\langle\bar{k}\rangle_{f, \infty}\left\langle\bar{E}^{m}\right\rangle_{f, \infty}}-\left(\frac{b}{\left\langle\bar{E}^{m}\right\rangle_{f, \infty}}-\frac{a-n}{\langle\bar{k}\rangle_{f, \infty}}\right)^{2}\right]^{1 / 2}$,

$\mathcal{A}=\frac{\left\langle\bar{E}^{m}\right\rangle_{f}(t=0)}{\left\langle\bar{E}^{m}\right\rangle_{f, \infty}}-1$.

For flows in stratified porous media, we observed that the oscillations vanish after one period (see Figs. 9-12). We thus impose:

$\frac{1}{\tau}=\omega$, which leads, using (54) and (55), to:

$b n=2 \omega^{2} \frac{\langle\bar{k}\rangle_{f, \infty}\left\langle\bar{E}^{m}\right\rangle_{f, \infty}}{P_{S F \infty}^{2}}$.

Moreover, reference calculations (see [4, p. 200]) show that $\mathbb{E}$ derivative vanishes for $t \simeq 14 D_{h} /\left\langle\bar{u}_{z}\right\rangle_{f}$ in plane channel and $t \simeq 19 D_{h} /\left\langle\bar{u}_{z}\right\rangle_{f}$ in circular pipe. According to (53), this yields:

$\frac{d \mathbb{E}}{d t}=0 \Longleftrightarrow \omega t=\frac{3 \pi}{4}[\pi]$.

Consequently, we find

$\omega=\frac{3 \pi}{56} \frac{\left\langle\bar{u}_{z}\right\rangle_{f}}{D_{h}}$, in plane channel,

$\omega=\frac{3 \pi}{76} \frac{\left\langle\bar{u}_{z}\right\rangle_{f}}{D_{h}}$, in circular pipe.

We also observed that $d \llbracket / d t$ vanishes for $t_{0} \simeq 24 D_{h} /\left\langle\bar{u}_{z}\right\rangle_{f}$ in plane channel and $t_{0} \simeq 34 D_{h} /\left\langle\bar{u}_{z}\right\rangle_{f}$ in circular pipe and Eq. (52) then gives

$\left(\frac{b}{\left\langle\bar{E}^{m}\right\rangle_{f, \infty}}+\frac{a-n}{\langle\bar{k}\rangle_{f, \infty}}\right)\left(\cos \omega t_{0}+\sin \omega t_{0}\right)=\frac{2 \omega}{P_{S F \infty}}\left(\sin \omega t_{0}-\cos \omega t_{0}\right)$.

Given the fact that sub-filter production is a transfer between dispersive and turbulent energies, we impose $a=b$. With this 


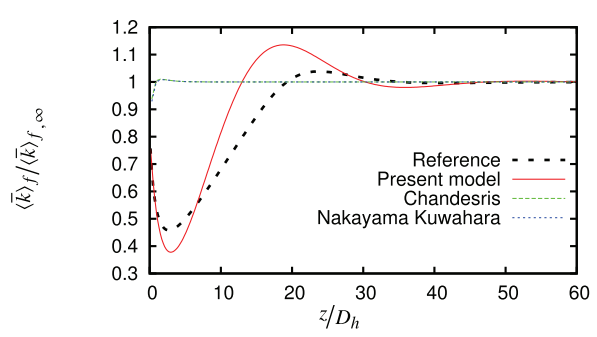

(a) Evolution of $\langle\bar{k}\rangle_{f} /\langle\bar{k}\rangle_{f, \infty}$

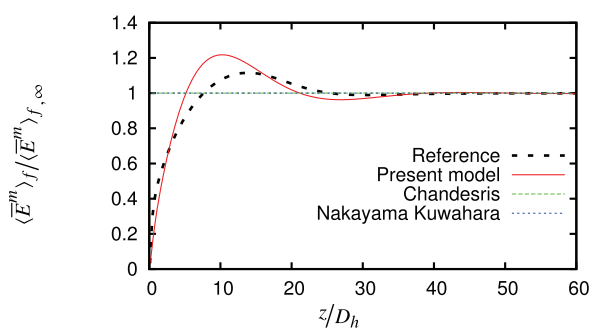

(c) Evolution of $\left\langle\bar{E}^{m}\right\rangle_{f} /\left\langle\bar{E}^{m}\right\rangle_{f, \infty}$

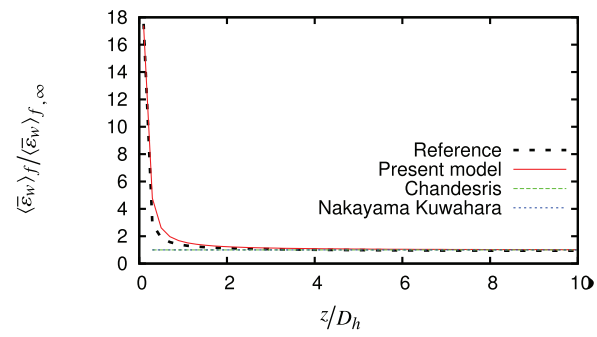

(e) Evolution of $\left\langle\bar{\varepsilon}_{w}\right\rangle_{f} /\left\langle\bar{\varepsilon}_{w}\right\rangle_{f, \infty}$

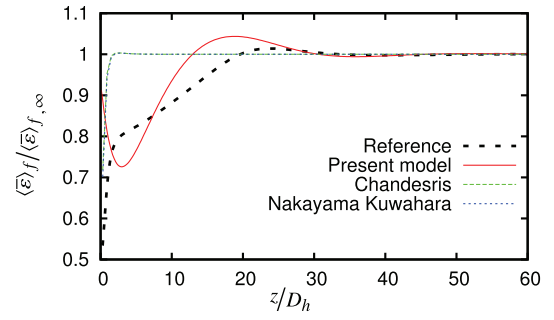

(b) Evolution of $\langle\bar{\varepsilon}\rangle_{f} /\langle\bar{\varepsilon}\rangle_{f, \infty}$

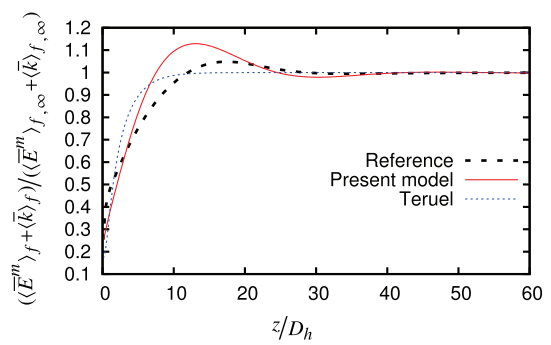

(d) Evolution of $\left(\left\langle\bar{E}^{m}\right\rangle_{f}+\langle\bar{k}\rangle_{f}\right) /\left(\left\langle\bar{E}^{m}\right\rangle_{f, \infty}+\langle\bar{k}\rangle_{f, \infty}\right)$

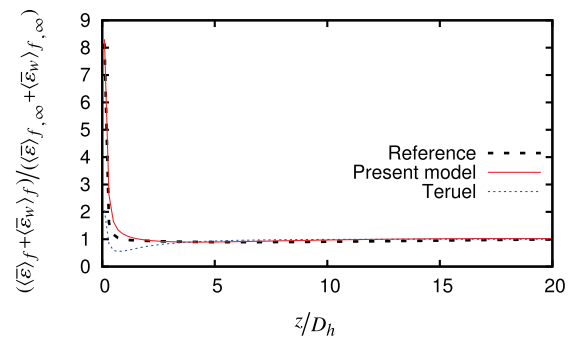

f) Evolution of $\left(\langle\bar{\varepsilon}\rangle_{f}+\left\langle\bar{\varepsilon}_{w}\right\rangle_{f}\right) /\left(\langle\bar{\varepsilon}\rangle_{f, \infty}+\left\langle\left\langle\bar{\varepsilon}_{w}\right\rangle_{f, \infty}\right)\right.$

Fig. 11. Macroscopic turbulence model results for turbulent flow in stratified porous media composed of flat plates, $R e=7.6 \times 10^{4}$; $\left\langle\bar{k}_{0}\right\rangle_{f} /\langle\bar{k}\rangle_{f, \infty}=0.8$. Comparison of the present model to reference results and to the models of Chandesris et al. [1], Nakayama and Kuwahara[13] and Teruel and Rizwan-uddin [22].

additional constraint, Eqs. (58), (60), (61) and (62) allow us to determine $n$ for several Reynolds numbers and the corresponding value of $a=b$.

The average values of the model constants for the test cases presented in Table 1 are: $a=b=1 / 4, n=1 / 3$. Sub-filter production and averaged viscous dissipation then read:

$P_{S F}=P_{S F \infty}\left(\frac{\langle\bar{k}\rangle_{f}}{\langle\bar{k}\rangle_{f, \infty}}\right)^{1 / 4}\left(\frac{\left\langle\bar{E}^{m}\right\rangle_{f}}{\left\langle\bar{E}^{m}\right\rangle_{f, \infty}}\right)^{1 / 4}$,

$\langle\bar{\varepsilon}\rangle_{f}=\langle\bar{\varepsilon}\rangle_{f, \infty}\left(\frac{\langle\bar{k}\rangle_{f}}{\langle\bar{k}\rangle_{f, \infty}}\right)^{1 / 3}$.

Eq. (32) is solved for $C_{\varepsilon_{w 1}} \in[0.1,10]$ and the results are compared with reference results. Optimal value for $C_{\varepsilon_{w, 1}}$ is then numerically found: $C_{\varepsilon_{w 1}}=1$. Calibration procedure is summarized if Table 2 . The macroscopic model finally reads, for turbulent flows in channels and pipes with no macroscopic shear:

$$
\begin{aligned}
\frac{\partial\langle\bar{k}\rangle_{f}}{\partial t}+\left\langle\bar{u}_{z}\right\rangle_{f} \frac{\partial}{\partial z}\langle\bar{k}\rangle_{f}= & \frac{\partial}{\partial z}\left[\left(v_{f}+\frac{v_{t \phi}}{\sigma_{k}}+\mathcal{D}_{z z}^{P}\right) \frac{\partial\langle\bar{k}\rangle_{f}}{\partial z}\right] \\
& +P_{S F}-\langle\bar{\varepsilon}\rangle_{f}, \\
\frac{\partial\left\langle\bar{E}^{m}\right\rangle_{f}}{\partial t}+\left\langle\bar{u}_{z}\right\rangle_{f} \frac{\partial}{\partial z}\left\langle\bar{E}^{m}\right\rangle_{f}= & \frac{\partial}{\partial z}\left[\left(v_{f}+\mathcal{D}_{z z}^{P}\right) \frac{\partial\left\langle\bar{E}^{m}\right\rangle_{f}}{\partial z}\right]-P_{S F} \\
& +\left\langle\bar{u}_{z}\right\rangle_{f} \bar{F}_{\phi_{z}}-\left\langle\bar{\varepsilon}_{w}\right\rangle_{f},
\end{aligned}
$$

$$
\begin{aligned}
\frac{\partial\left\langle\bar{\varepsilon}_{w}\right\rangle_{f}}{\partial t} & +\left\langle\bar{u}_{z}\right\rangle_{f} \frac{\partial}{\partial z}\left\langle\bar{\varepsilon}_{w}\right\rangle_{f}=\frac{\partial}{\partial z}\left[\left(v_{f}+\mathcal{D}_{z z}^{P}\right) \frac{\partial\left\langle\bar{\varepsilon}_{w}\right\rangle_{f}}{\partial z}\right] \\
& +C_{\varepsilon_{w, 1}}\left\langle\bar{u}_{z}\right\rangle_{f} \frac{\left\langle\bar{\varepsilon}_{w}\right\rangle_{f}}{\left\langle\bar{E}^{m}\right\rangle_{f}}\left(\bar{F}_{\phi_{z}}-\bar{F}_{\phi_{z}}^{\infty} \frac{\left\langle\bar{\varepsilon}_{w}\right\rangle_{f}}{\left\langle\bar{\varepsilon}_{w}\right\rangle_{f, \infty}}\right),
\end{aligned}
$$

with the following closure relationships:

$$
\begin{aligned}
& f_{w}=f_{w \infty}\left(\frac{\left\langle\bar{\varepsilon}_{w}\right\rangle_{f}}{\left\langle\bar{\varepsilon}_{w}\right\rangle_{f, \infty}}\right)^{2 / 3} \\
& P_{S F}=P_{S F \infty}\left(\frac{\langle\bar{k}\rangle_{f}}{\langle\bar{k}\rangle_{f, \infty}}\right)^{1 / 4}\left(\frac{\left\langle\bar{E}^{m}\right\rangle_{f}}{\left\langle\bar{E}^{m}\right\rangle_{f, \infty}}\right)^{1 / 4}, \\
& \langle\bar{\varepsilon}\rangle_{f}=\langle\bar{\varepsilon}\rangle_{f, \infty}\left(\frac{\langle\bar{k}\rangle_{f}}{\langle\bar{k}\rangle_{f, \infty}}\right)^{1 / 3}
\end{aligned}
$$

$\left\langle\bar{u}_{z}\right\rangle_{f} \bar{F}_{\phi_{z}}=\frac{\left\langle\bar{u}_{z}\right\rangle_{f}^{3}}{2 D_{h}} f_{w}$,

$v_{t \phi}=C_{\mu}^{\prime} \frac{\langle\bar{k}\rangle_{f}^{2}}{\langle\bar{\varepsilon}\rangle_{f}}$,

$\mathcal{D}_{z z}^{P}=\frac{C_{\mu}^{\prime \prime}}{\operatorname{Pr}_{d}} \frac{\left\langle\bar{E}^{m}\right\rangle_{f}}{\left\langle\bar{\varepsilon}_{w}\right\rangle_{f}}$, 


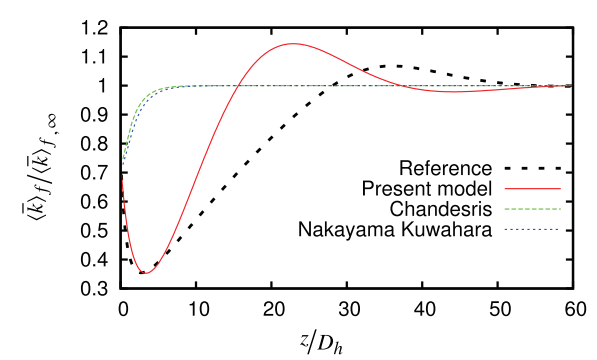

(a) Evolution of $\langle\bar{k}\rangle_{f} /\langle\bar{k}\rangle_{f, \infty}$

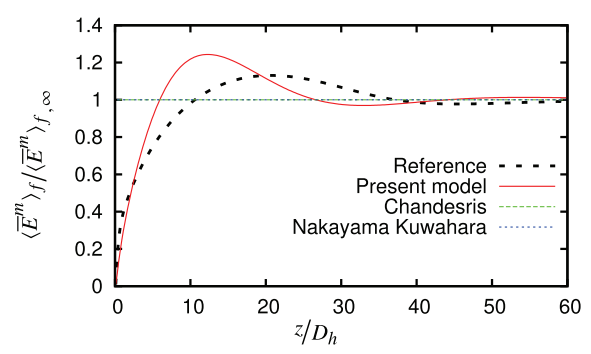

(c) Evolution of $\left\langle\bar{E}^{m}\right\rangle_{f} /\left\langle\bar{E}^{m}\right\rangle_{f, \infty}$

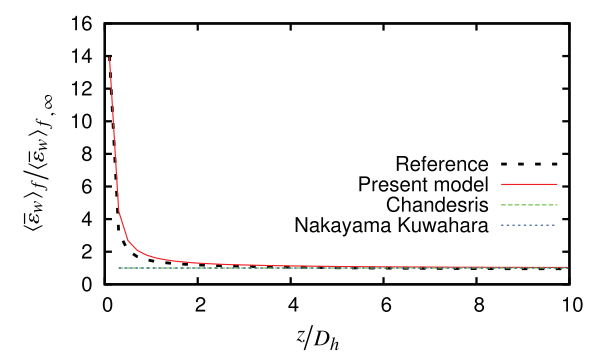

(e) Evolution of $\left\langle\bar{\varepsilon}_{w}\right\rangle_{f} /\left\langle\bar{\varepsilon}_{w}\right\rangle_{f, \infty}$

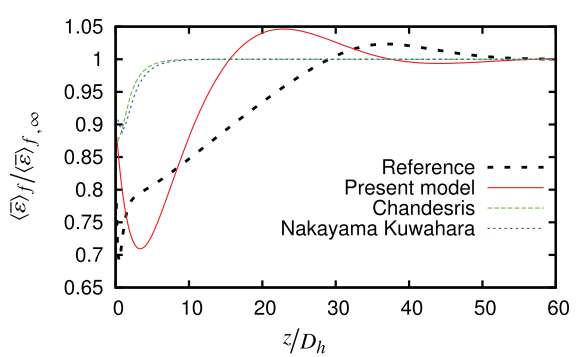

(b) Evolution of $\langle\bar{\varepsilon}\rangle_{f} /\langle\bar{\varepsilon}\rangle_{f, \infty}$

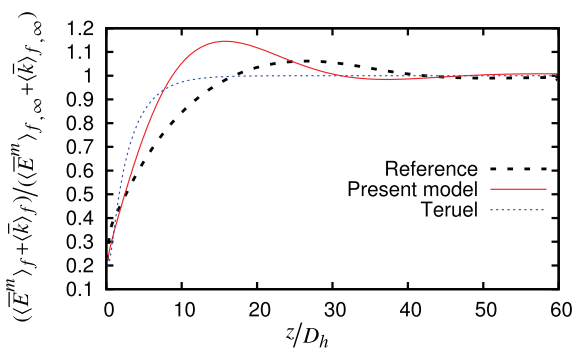

(d) Evolution of $\left(\left\langle\bar{E}^{m}\right\rangle_{f}+\langle\bar{k}\rangle_{f}\right) /\left(\left\langle\bar{E}^{m}\right\rangle_{f, \infty}+\langle\bar{k}\rangle_{f, \infty}\right)$

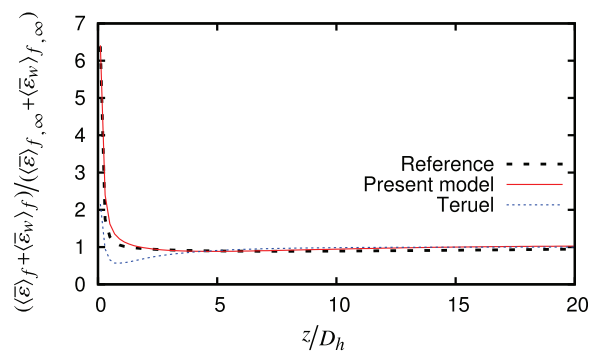

(f) Evolution of $\left(\langle\bar{\varepsilon}\rangle_{f}+\left\langle\bar{\varepsilon}_{w}\right\rangle_{f}\right) /\left(\langle\bar{\varepsilon}\rangle_{f, \infty}+\left\langle\bar{\varepsilon}_{w}\right\rangle_{f, \infty}\right)$

Fig. 12. Macroscopic turbulence model results for turbulent flow in stratified porous media composed of circular pipes, $R e=7.6 \times 10^{4} ;\left\langle\bar{k}_{0}\right\rangle_{f} /\langle\bar{k}\rangle_{f, \infty}=0.8$. Comparison of the present model to reference results and to the models of Chandesris et al. [1], Nakayama and Kuwahara[13] and [22].

Table 1

Description of the test cases used to determine the model constants. All cases are simulated for plane channel and circular pipe geometries.

\begin{tabular}{lll}
\hline Nom & $R e$ & $\left\langle\bar{k}_{0}\right\rangle_{f} /\langle\bar{k}\rangle_{f, \infty}$ \\
\hline SIM_REF_1 & $1 \times 10^{5}$ & 3 \\
SIM_REF_2 & $1 \times 10^{5}$ & 3 \\
SIM_REF_3 & $1 \times 10^{5}$ & $1 / 2$ \\
SIM_REF_4 & $5 \times 10^{4}$ & 3 \\
\hline
\end{tabular}

Table 2

Summary of the calibration procedure.

\begin{tabular}{lll}
\hline Coefficient & Calibration procedure & Value \\
\hline$C_{w}$ & Asymptotic state & 3 \\
$C_{k}$ & Asymptotic state & 1.82 for plane channels and 2.32 \\
& & for pipes \\
$C_{\varepsilon_{w, 1}}$ & Dynamical behavior & 1 \\
$a=b$ & Dynamical behavior & $1 / 4$ \\
$n$ & Dynamical behavior & $1 / 3$ \\
$C_{\mu}^{\prime}$ & Same value as $k-\bar{\varepsilon}$ model & 0.09 \\
$C_{\mu}^{\prime \prime} / P r_{d}$ & Best fit of the passive & 5.85 \\
& dispersion model [5] & \\
$C_{t}^{A}$ & Active dispersion model [5] & 1.63 for plane channels and 2.1 \\
$\mathcal{B}$ & for $P r=1$ & for circular pipes \\
& Active dispersion model [5] & 3692 for plane channels and 2623 \\
& for $P r=1$ & for circular pipes \\
\hline
\end{tabular}

$\mathcal{D}_{z}^{A^{*}}=\mathcal{C}_{t}^{A}+\frac{\mathcal{B}}{\sqrt{f_{w}} R e}$,

and the asymptotic state values:

$\langle\bar{k}\rangle_{f, \infty}=c_{k} u_{\tau}^{2}$,

$\left\langle\bar{\varepsilon}_{w}\right\rangle_{f, \infty}=C_{w} \frac{\left\langle\bar{u}_{z}\right\rangle_{f}^{3}}{2 D_{h}} f_{w \infty}^{3 / 2}$

$\left\langle\bar{E}^{m}\right\rangle_{f, \infty}=2 \mathcal{D}_{z}^{A^{*}} u_{\tau}^{2}$

$P_{S F \infty}=\langle\bar{\varepsilon}\rangle_{f, \infty}=\frac{\left\langle\bar{u}_{z}\right\rangle_{f}^{3}}{2 D_{h}} f_{w \infty}\left(1-C_{w} f_{w \infty}^{1 / 2}\right)$.

Calibration procedure for the phenomenological constants is summarized in Table 2.

\section{Assessment of the macroscopic turbulence model}

In order to assess the present macroscopic model, steady unidirectional turbulent flows entering porous media composed of plane channels and circular pipes are investigated from both microscopic and macroscopic points of view. From the microscopic point of view, fine-scale simulations are carried out with FLICA-OVAP CFD code [6] using the low-Reynolds $\bar{k}-\bar{\varepsilon}$ [3] model. Simulations results thus produced are spatially averaged and are 
used as reference results. At the inlet, uniform velocity, turbulent kinetic energy and viscous dissipation profiles are imposed. Spatially averaged quantities at the inlet provide channel inlet boundary conditions hereafter denoted ".o". Fine scale simulation results are similar to the results of [19] even though we use different RANS model and computational code. In particular, between inlet and asymptotic state, large scale oscillations of spatially averaged physical quantities are observed.

From the macroscopic point of view, Eqs. (34)-(36) are solved with the closure relationships (33), (29) and (30). The macroscopic turbulence model has been implemented in a 1D code with a numerical scheme based upon MUSCL formulation. Calculation results are all fully spatially converged. Asymptotic states are given by Eqs. (37), (38), (40) and (45). Three other macroscopic turbulence models: Chandesris et al. [1], Nakayama and Kuwahara [13] and Teruel and Rizwan-uddin [22] have also been implemented following the same discretization and numerical scheme.

In this paper we both investigate the influence of the inlet turbulence intensity and of the underlying geometry on model prediction capabilities. Two type of "configurations" are presented hereafter, for both plane channel and circular pipe geometries:

1. the "strong" turbulence, characterized by a turbulence level at the inlet much higher than the asymptotic value of the medium $\left(\left\langle\bar{k}_{0}\right\rangle_{f} /\langle\bar{k}\rangle_{f, \infty}=4\right)$,

2. the "soft" turbulence, that exhibits a turbulence level lower to its asymptotic value $\left(\left\langle\bar{k}_{0}\right\rangle_{f} /\langle\bar{k}\rangle_{f, \infty}=0.8\right)$.

Inlet value of $\bar{E}^{m}$ is zero since there is no velocity deviation. The inlet values for $\bar{\varepsilon}_{w}$ come from spatially averaged fine scale calculation results. In the future, this quantity shall be connected to singular pressure loss models. The same bulk Reynolds number is used for both geometries $(R e=76000)$. Results of the present model are compared with spatially averaged fine-scale simulations in Figs. 9-12. Agreement between reference results and our macroscopic model is good. Better results are obtained for strong turbulence intensity at the inlet. Obviously, asymptotic regime is recovered and the dynamical behavior of major quantities in the inlet region agrees well with reference results for amplitude of oscillations and damping length. As $\left\langle\bar{\varepsilon}_{w}\right\rangle_{f}$ is well predicted, even in the inlet region, this means according to (68) that friction factor is also well predicted.

Figs. 9-12 also present a comparison between the present model and the models of Chandesris et al. [1], Nakayama and Kuwahara [13] and Teruel and Rizwan-uddin [22] adapted to pipe flows. One can see that our model gives much better results than the other ones, especially for the dynamical behavior. Indeed, by taking into account the evolutions of sub-filter mean kinetic energy and friction coefficient, we strongly improve the ability of the macroscopic turbulence model to reproduce the establishment of the velocity profiles, and consequently the general dynamic behavior of the flow. Other models mainly relax form inlet conditions to asymptotic values. Let us note that the capability of the proposed model to account for Reynolds number variations is exposed in [4].

\section{Conclusion}

A macroscopic turbulence model, based on a two-scale analysis, and a calibration methodology have been proposed for flows in porous media. The two-scale analysis highlighted energy transfers between the mean motion and turbulence embedded in a porous medium. Averaged energies equations have been derived and closure relationships have been determined for flows in channels. The present model also accounts for the dispersive energy and can thus be used to derive a general model connecting macroscale turbulence and thermal dispersion modeling. The general calibration methodology exposed here allows the model to both recover relevant asymptotic values and dynamical behavior when submitted to perturbations. Models for asymptotic values may be used to feed inlet boundary conditions for calculations that focus on the downstream region of the porous medium. The model have been extensively tested for unidirectional turbulent flows in stratified porous media. Reference results are accurately recovered, as well as asymptotic values. Moreover, comparisons with results provided by Nakayama and Kuwahara [13], Chandesris et al. [1], and Teruel and Rizwan-uddin [22] show significant improvements. A natural extension of this model shall aim to couple it with the advanced heat exchange model exposed in Grégoire et al. [9].

\section{References}

[1] M. Chandesris, G. Serre, P. Sagaut, A macroscopic model for flows in porous media suited for channel, pipe and rod bundle flows, Int. J. Heat Mass Transfer 49 (2006) 2739-2750.

[2] M. Chandesris, Modélisation des écoulements turbulents dans les milieux poreux et à l'interface avec un milieu libre, Thèse de Doctorat de l'Université Paris VI, 2006.

[3] K.Y. Chien, Predictions of channel and boundary layer flows with a low Reynolds number turbulence model, AIAA J. 20 (1982) 33-38.

[4] M. Drouin, Modélisation des écoulements turbulents anisothermes en milieux macroporeux par une approche de double filtrage, Ph.D. Thesis, INP Toulouse, 2010.

[5] M. Drouin, O. Grégoire, O. Simonin, A. Chanoine, Macroscopic modeling of thermal dispersion for turbulent flows in channels, Int. J. Heat Mass Transfer 53 (2010) 2206-2217.

[6] P. Fillion, A. Chanoine, S. Dellacherie, A. Kumbaro, FLICA-OVAP: a new platform for core thermal-hydraulic studies, in: NURETH-13, 2009.

[7] J.J. Finnigan, Turbulence in plant canopy, Annu. Rev. Fluid Mech. 32 (2000) 519-571.

[8] S.R. Green, Modelling turbulent air flow in a stand of widely-spaced trees, PHOENICS J. Comput. Fluid Dyn. Appl. 5 (1992) 194-312.

[9] O. Grégoire, M. Drouin, O. Simonin, Connecting dispersion models and wall temperature prediction for laminar and turbulent flows in channels, Int. J. Heat Mass Transfer 55 (2012) 3100-3113.

[10] S. Kakaç et al., Handbook of Single-phase Convective Heat Transfer, John Wiley and Sons, 1987.

[11] T. Masuoka, Y. Takatsu, Turbulence model for flow through porous media, Int. J. Heat Mass Transfer 39 (1996) 2803-2809.

[12] A. Nakayama, F. Kuwahara, A macroscale turbulence model for flows in a porous medium, J. Fluids Eng. 121 (1999) 427-433.

[13] A. Nakayama, F. Kuwahara, A general macroscopic turbulence model for flows in packed beds, channels, pipes and rod bundles, J. Fluids Eng. 130 (2008) 101205.

[14] D.A. Nield, Alternative models of turbulence in a porous medium and related matters, J. Fluids Eng. 123 (2001) 928-931.

[15] M.H.J. Pedras, M.J.S. de Lemos, On the definition of turbulent kinetic energy for flow in porous media, Int. Commun. Heat Mass Transfer 27 (2000) 211-220.

[16] M.H.J. Pedras, M.J.S. de Lemos, Macroscopic turbulence modeling for incompressible flow through undeformable porous media, Int. J. Heat Mass Transfer 44 (2001) 1081-1093.

[17] F. Pinson, Modélisation à l'échelle macroscopique d'un écoulement turbulent au sein d'un milieu poreux, Ph.D. Thesis, INP Toulouse, 2006.

[18] F. Pinson, O. Grégoire, O. Simonin, $k-\varepsilon$ Macro-scale modeling of turbulence based on a two scale analysis in porous media, Int. J. Heat Fluid Flow 27 (2006) 955-966.

[19] F. Pinson, O. Grégoire, O. Simonin, Macro-scale turbulence modeling for flows in media laden with solid structure, C.R. Méc. 335 (2007) 13-19.

[20] M. Quintard, S. Whitaker, Transport in ordered and disordered porous media I: the cellular average and the use of weighting functions, Transp. Porous Media 14 (1994) 163-177.

[21] F.E. Teruel, Rizwan-uddin, A new turbulence model for porous media flows. Part I: constitutive equations and model closure, Int. J. Heat Mass Transfer 52 (2009) 79-90.

[22] F.E. Teruel, Rizwan-uddin, A new turbulence model for porous media flows. Part II: analysis and validation using microscopic simulations, Int. J. Heat Mass Transfer 52 (2009) 4264-4272.

[23] V.S. Travkin, Discussion: alternative models of turbulence in a porous medium and related matters, J. Fluid Eng. - Trans. ASME 123 (2001) 931-934.

[24] S. Whitaker, Diffusion and dispersion in porous media, AIChE 3 (13) (1967) 420-427. 